\title{
Selective endocytosis controls slit diaphragm maintenance and dynamics.
}

Konrad Lang1, Julian Milosavljevic ${ }^{1}$, Helena Heinkele ${ }^{1}$, Mengmeng Chen ${ }^{1}$, Lea Gerstner', Dominik Spitz', Séverine Kayser ${ }^{1}$, Martin Helmstädter ${ }^{1}$, Gerd Walz ${ }^{1,2}$, Michael Köttgen ${ }^{1,2}$, Andrew Spracklen ${ }^{3}$, John Poulton ${ }^{4}$, and Tobias Hermle ${ }^{1^{*}}$

1 Renal Division, Department of Medicine, Faculty of Medicine and Medical Center - University of Freiburg, Freiburg, Germany

2 CIBSS - Centre for Integrative Biological Signalling Studies, Freiburg, Germany

3 Lineberger Comprehensive Cancer Center, University of North Carolina at Chapel Hill, North Carolina, USA. Present address: Department of Biology, University of Massachusetts, Amherst, USA

$4 \quad$ Department of Medicine, Division of Nephrology and Hypertension, University of North Carolina at Chapel Hill, North Carolina, USA

Running title: Renal filter needs selective endocytosis

\section{${ }^{*}$ Correspondence:}

Dr. Tobias Hermle

Renal Division, Department of Medicine

University Medical Center Freiburg

Hugstetter Strasse 55

79106 Freiburg, Germany

Email: tobias.hermle@uniklinik-freiburg.de 


\section{SUMMARY}

The kidneys generate about 180 liters of primary urine per day by filtration of plasma. An essential part of the filtration barrier is the slit diaphragm, a multiprotein complex containing nephrin as major component. Filter dysfunction typically manifests with proteinuria and mutations in endocytosis regulating genes were discovered as causes of proteinuria. However, it is unclear how endocytosis regulates the slit diaphragm and how the filtration barrier is maintained without either protein leakage or filter clogging. Here we study nephrin dynamics in podocyte-like nephrocytes of Drosophila and show that selective endocytosis either by dynamin- or flotillin-mediated pathways regulates a stable yet highly dynamic architecture. Short-term manipulation of endocytic functions indicates that dynamin-mediated endocytosis of ectopic nephrin restricts slit diaphragm formation spatially while flotillin-mediated turnover of nephrin within the slit diaphragm is needed to maintain filter permeability by shedding of molecules bound to nephrin in endosomes. Since slit diaphragms cannot be studied in vitro and are poorly accessible in mouse models, this is the first analysis of their dynamics within the slit diaphragm multiprotein complex. Identification of the mechanisms of slit diaphragm maintenance will help to develop novel therapies for proteinuric renal diseases that are frequently limited to symptomatic treatment. 


\section{INTRODUCTION}

The human kidneys maintain water and electrolyte homeostasis and efficiently excrete metabolic waste products and xenobiotics. The essential first step of kidney function is to generate primary urine by filtration of blood across a size- and charge-selective filter. Every single day, the kidneys are perfused with 1700 liters of blood and filter about 180 liters of nearly protein-free of primary urine thus retaining approximately $12 \mathrm{~kg}$ of plasma protein from the filtered fraction. It remains unclear, how it is possible to maintain this filter during constant filtration without leakage of plasma protein or clogging while adapting to changing physiological conditions ${ }^{1,2}$.

The filtration barrier is provided by two epithelial layers, the vascular endothelium and the glomerular podocytes with their interjacent basement membrane. The filtrate traverses through endothelial pores, the basement membrane and narrow filtration slits that form between the elaborate network of the podocytes' interdigitating foot processes ${ }^{1}$. These slits are guarded by the slit diaphragm whose major structural components are nephrin and NEPH1 that engage transcellularly ${ }^{3-7}$. However, slit diaphragms represent a multiprotein complex that includes further proteins such as podocin ${ }^{8,9}$ and associates with proteins like TRPC6 $6^{10,11}$ to direct signaling ${ }^{12,13}$. Several lines of evidence support a role of endocytic pathways for proper function of the filtration barrier ${ }^{14}$. Overexpressed nephrin is subject to endocytosis in vitro ${ }^{15,16}$ and dysregulation of endocytosis in murine podocytes resulted in severe proteinuria, the clinical hallmark of a failing glomerular filter ${ }^{17-19}$. In mice, PKC- $\alpha$ and CIN85 promote nephrin endocytosis under diabetic conditions and similarly after Angiotensin II exposure ${ }^{20-}$ 23. We and others discovered monogenic mutations of endosomal regulators as the molecular cause of severe proteinuria in humans ${ }^{24-26}$. Surprisingly, mutations of these widely-expressed genes exclusively manifested with nephrotic syndrome ${ }^{24-26}$. While endocytosis occurs ceaselessly in all cells, the kidney's filtration barrier thus requires a particularly tight regulation of endocytic trafficking. Endocytosis might be needed for slit diaphragm formation, renewal and/or restriction of slit diaphragms to their proper location. Elucidating these fundamental aspect of podocyte biology represents a major challenge due the rapid transport dynamics and complex architecture of the filtration barrier. Overexpression of nephrin in immortalized cells rendered significant insights, but the lack of slit diaphragms in in vitro models entails that nephrin is not embedded within a proper multiprotein complex. Genetic mouse models allowed identification of essential genes but they cannot provide insights into dynamic remodeling/recycling of the slit diaphragm due to slow throughput and limited accessibility. Thus, we employed the podocyte-like nephrocytes in Drosophila 
that form functional slit diaphragms using orthologous proteins ${ }^{27-30}$. Utilizing this model, we developed assays to examine slit diaphragm dynamics directly after short-term manipulation of endocytic functions and obtained unique in vivo insights into the filtration barrier's dynamics. Lateral diffusion of ectopic nephrin is prevented by rapid dynamin-dependent endocytosis restricting slit diaphragm localization. In contrast, nephrin engaged within the proper slit diaphragm complex is constantly endocytosed flotillin2-dependently followed by recycling. Such turnover offers flexibility and cleanses the filtration barrier from adherent molecules, maintaining its permeability. Selective and functionally distinct routes of endocytic transport thus maintain barrier's architecture and permeability. 


\section{RESULTS}

\section{Slit diaphragms in nephrocytes are stable structures that dynamically reconstitute upon disruption.}

The podocyte-like nephrocytes form functional slit diaphragms that filter larval plasma before entry into membrane invaginations termed labyrinthine channels (schematic Fig. 1A). The Drosophila slit diaphragm proteins Sns and Kirre are orthologous to human nephrin and NEPH1 and engage in a multiprotein complex ${ }^{29,31,32}$. For simplicity, we will use the human names for the Drosophila orthologs throughout the manuscript. As a consequence of the nephrocyte's cytoarchitecture, these proteins stain in a linear pattern reminiscent of fingerprints in tangential sections and adhere to the cell membrane in a dotted line in cross sections ${ }^{29}$ (Fig. 1A-B, Suppl. Fig. 1A-A"). To explore slit diaphragm dynamics, we introduced GFP into the C-terminus of the endogenous nephrin (sns) locus via genome editing (schematic of slit diaphragm with tag Fig. 1B). Genomic nephrin-GFP resulted in expression of functional protein that sustained regular slit diaphragms in a homozygous state (Fig. 1B electron micrograph). Nephrin-GFP colocalized with endogenous Neph1 (Fig. 1B bottom panels, Suppl. Fig. 1B-C), suggesting integration into the slit diaphragm. Confirming its specificity, the GFPderived fluorescence was abrogated by nephrin silencing (Suppl. Fig. 1D-D'). Using this model, we studied slit diaphragm dynamics by live-cell imaging ex vivo. We observed a stable slit diaphragm architecture over a period of up to one hour (Fig. 1C-D, Suppl. videos 1-2). To explore the half-life of wild-type nephrin, we employed a temperature-sensitve GAL80 to modify GAL4-dependent transgene expression by temperature shifts (active at $31{ }^{\circ} \mathrm{C}$, inactive at $18{ }^{\circ} \mathrm{C}$ ). Upon short-term expression of nephrin-RNAi, we observed an incremental loss of nephrin protein, as indicated by shorter slit diaphragm lines. A reduction of approximately $50 \%$ in length and density was reached after nephrin silencing for 2 days (compare Fig. 1E-E”' and Fig. 1F-F”, silencing for 24 h Suppl. Fig. 1E-E'”). This implies an extensive half-life for nephrin protein ranging from 1-3 days. To investigate if slit diaphragms may reconstitute after disruption, we used GAL80ts/GAL4 to first silence nephrin (Fig. 1G-G”) before stopping RNAi expression, which resulted in slow return of nephrin after three to four days. Few isolated slit diaphragms were detectable in electron microscopy at that stage (inset Fig. 1H'). In confocal microscopy the lines of slit diaphragms elongated over time (Fig. 1H, additional images Suppl. Fig. 1G-J'), and gradually repopulated the nephrocyte's surface, frequently in pairs (Fig. 1I, live-cell imaging Suppl. Fig. 1J-J", Suppl. Video 3), and finally restored normal density 
(Fig. 1J-J"). Slit diaphragms thus are formed by a protein with an extensive half-life and may reconstitute after disruption.

\section{Live antibody labeling and FRAP suggest rapid slit diaphragm turnover.}

We hypothesized that slit diaphragms are subject to endocytic turnover in vivo as previously suggested by in vitro studies ${ }^{14}$. To study the dynamics of nephrin within the slit diaphragm, we introduced a Myc-tag into the extracellular domain of nephrin by CRISPR-editing the second exon (Suppl. Fig. 2A). Myc-staining revealed a typical slit diaphragm pattern, colocalizing with Neph1 (Fig. 2A, Suppl. Fig. 2B-C'”). The Myc-signal was lost upon nephrin silencing (Suppl. Fig. 2D-D'), and homozygous animals formed regular slit diaphragms (Fig. 2B). This indicates that a functional fusion protein is expressed from the edited locus. The extracellular tag was labeled ex vivo by exposing living nephrocytes to anti-Myc antibody. We tracked the fate of the live antibody-labeled nephrin protein by further incubating the living cells (chase period). After fixation and permeabilization, regular Myc-staining was employed to detect the entire nephrin protein (schematic Fig. 2C). Without chasing, the live labeled antibody matched the pattern obtained by the subsequent total stain (Fig. 2D-D”), which confirms efficient live labeling. With progressive incubation time, the signal from live labeling at the slit diaphragms decreased, while a faint, diffuse intracellular signal appeared (Fig. 2E-F'). Residual signal of live labeled antibody at the slit diaphragm persisting even after 2 hours suggested a small immobile fraction. At the end of chasing, we further detected a slit diaphragm pattern that was exclusively derived from the total staining while largely lacking in live labeling (Fig. 2F-F'). This indicates that during the chase period new protein had reached intact slit diaphragms. Quantification of the surface-derived Myc-nephrin signal in ratio to the submembraneous intracellular signal indicated steady reduction over time, supporting a constant endocytic turnover (Fig. 2G, Suppl. Fig. 2E). To evaluate this turnover independently, we employed the CRISPR-edited nephrin-GFP to perform Fluorescence Recovery After Photobleaching (FRAP) experiments (Fig. 2H, quantitation Fig. 2I). Confirming our findings with live antibody labeling, FRAP analysis indicated a rapid recovery after bleaching with $50 \%$ recovery of the signal after only $\sim 7 \mathrm{~min}$. The recovery reached a plateau within 30 min, suggesting an immobile fraction of fly nephrin that is not replaced by turnover during the observation period. However, the majority of nephrin protein undergoes quite rapid cycles of turnvover.

\section{Rab5 regulates trafficking of fly nephrin.}


We now wanted to explore how manipulation of the endocytic activity affects nephrin. The most basic steps of endocytosis are uptake followed by sorting either towards degradation or recycling to the plasma membrane. The small GTPase Rab5 localizes to early endosomes, where it regulates uptake, endosomal fusion and cargo sorting (schematic Fig. 3A). We overexpressed the constitutively active $R a b 5^{Q 88 L}$ in nephrocytes limiting expression to $24 \mathrm{~h}$ using $G A L 80^{t s}$ to avoid non-specific effects. YFPRab5Q88L induced enlarged endosomes containing nephrin (Fig. 3B-B', control Suppl. Fig. 3A-B'). This indicated that increased Rab5 function redirected nephrin to endosomes. Subsequently, we evaluated short-term silencing of Rab5 for $17 \mathrm{~h}$. This time sufficed to extensively reduce Rab5 protein (Suppl. Fig. 3C-D), without affecting cellular viability since nephrocytes remained negative for cell death marker terminal deoxynucleotidyl transferase dUTP nick end labeling (TUNEL, Suppl. Fig. 3EE', positive control Suppl. Fig.3F-F'). In this early phase of disrupted endocytosis, the lines of slit diaphragm proteins became blurry and began to fuse (Fig. 3C). In cross sections, we observed extensive translocation of nephrin from the cell surface deeper into the cell (Fig. 3D). After prolonged Rab5 silencing for $24 \mathrm{~h}$, we observed a localized breakdown of slit diaphrams on sections of the cell surface (Fig. 3E-F''). This was matched by gradual expansion of slit diaphragm gaps in live imaging (Fig. 3G-G", Suppl. Video 4). To confirm a Rab5-specific effect, we employed dominant negative Rab5 $5^{S 4 N}$, which phenocopied our findings using Rab5-RNAi (Suppl. Fig. 3G-H'). Rab5 disruption thus has a severe impact on slit diaphragm maintenance. To correlate the subcortical nephrin with potential aberrant endosomes, we exposed nephrocytes during acute silencing of Rab5 to an extended course of tracer FITC-albumin which is rapidly endocytosed by nephrocytes ${ }^{29}$. Despite partial silencing of Rab5, we observed significant tracer endocytosis under these conditions (Fig. $\mathbf{3 H}$ H'). While this observation confirmed preserved cell viability and residual endocytic activity, we did not observe colocalization of the endocytic tracer and subcortical nephrin. Nephrin thus translocates extensively to an ectopic location that differs from an (early) endosomal compartment.

\section{Rab5 silencing causes lateral diffusion of slit diaphragm proteins and alters filtration characteristics.}

We hypothesized that lateral diffusion of slit diaphragm proteins into labyrinthine channels contributes to intracellular translocation of nephrin during acute silencing of Rab5. To simultaneously visualize the nephrocytes' labyrinthine channels and nephrin, we filled these invaginations via passive diffusion by incubating nephrocytes in Texas-Red-dextran (10 kDa) after brief fixation before staining nephrin (Fig. 4A). This approach reflected normal channel morphology in control cells (Fig. 4B), as well as 
the expected loss of the invaginations upon nephrin silencing (Suppl. Fig. 4A-A"). While nephrin was absent from the membrane invaginations under control conditions (Fig. 4B), we observed partial colocalization of ectopic nephrin with the channels in Rab5-RNAi nephrocytes (Fig. 4C). This suggested that nephrin partially translocated to the membrane invaginations upon disruption of endocytosis. Live imaging showed increasing formation of clusters of nephrin-GFP below the cell which preceded the localized breakdown (Fig. 4D, Suppl. Video 4) Live imaging further indicated dynamic movement of subcortical nephrin, likely caused by moving labyrinthine channels. Nephrin was removed in vesicles, suggesting residual, but misdirected endocytosis (Fig. 4E, Suppl. Video 5). Electron microscopy uncovered slit diaphragms deeply within the labyrinthine channels often in rosette-like clusters upon acute silencing of Rab5 (Fig. 4G, control Fig. 4F). This further supports lateral diffusion of nephrin protein, likely due to insufficient removal of the ectopic nephrin caused by impaired endocytosis.

To evaluate if Rab5-RNAi alters nephrocyte filtration barrier permeability, we recorded simultaneous endocytosis of tracers FITC-albumin $(66 \mathrm{kDa})$ that is close to the filtration barrier's size limit for passage $^{29}$ and the considerably smaller tracer Texas-Red-dextran (10 kDa). In nephrocytes expressing Rab5-RNAi, the decrease in uptake of FITC-albumin was about twice as strong as reduction of the smaller Texas-Red-dextran (Fig. $4 \mathbf{H}-\mathbf{I}$ ). In contrast, nephrin silencing reduced uptake of both tracers equally (Fig. 4J-J'"). Accordingly, the ratio of the fluorescence of the small tracer relative to the large tracer was strongly elevated for Rab5, while the ratio was unchanged by nephrin silencing (Fig. 4K). This observation suggests a reduced permeability of the slit diaphragm for larger tracer following disruption of endocytosis (Fig. 4L). We simultaneously exposed nephrocytes to another pair of tracers (Texas Red-Avidin, $66 \mathrm{kDa}$, and Alexa488 wheat germ agglutinin, $38 \mathrm{kDa}$ ) and Rab5-RNAi in turn affected uptake of the larger tracer more severely (Suppl. Fig. 4B-D). Comparing the rate of passive diffusion of FITC-albumin and Texas-Red-dextran (10 kDa) across the slit diaphragm into labyrinthine channels after brief fixation of nephrocytes similarly indicated reduced penetrance of the larger tracer (Suppl. Fig. 4E-G). Taken together, we conclude that defective endocytosis alters permeability of the nephrocyte's slit diaphragm in a size-dependent manner, suggesting incipient filter clogging.

\section{Slit diaphragm maintenance requires endocytosis and recycling but not degradation.}

To explore the contribution of key aspects endocytic cargo processing, we studied the effect of silencing critical Rab proteins. Expression of Rab7-RNAi, directed against the major Rab GTPase 
promoting degradation, or expression of dominant negative Rab7 had no overt effect on the slit diaphragm architecture (Fig. 5A-A', Suppl. Fig. 5A-A", Rab7 staining of control vs. knockdown Suppl. Fig. 5B-C'). However, Rab7-RNAi caused an additional faint nephrin signal in the cell (Fig. 5A-A", Suppl. Fig. 5A-A", Suppl. Fig. 5C-C"), suggesting accumulation of endocytosed nephrin protein due to lack of degradation. This further suggests that the vast majority of endocytosed nephrin returns to the plasma membrane through recycling pathways. EM also revealed accumulation of vesicles with lipid-like electron-density upon expression of Rab7-RNAi (Fig. 5B). We previously observed progressive loss of nephrin after acute silencing of Rab1126. We confirmed a role for recycling using a second Rab11-RNAi, observing coarser and wider spaced slit diaphragms (Suppl. Fig. 5D-E'), with nephrin and Neph1 appearing independently on the cell surface (insets in Suppl. Fig. 5E-E'", Rab11 staining of control vs. knockdown Suppl. Fig. 5F-G). Compensatory transport through alternative pathways such as Rab4-mediated recycling thus may be less effective in maintaining the slit diaphragm proteins in their stoichiometry and coherence during transport. Ultrastructural analysis upon silencing of Rab11 revealed formation of multiple slits within shortened labyrinthine channels and further excessive formation of lysosomes (red asterisks, Fig. 5C). As all steps of endocytosis are connected, this led us to hypothesize that lack of recycling intensifies degradation but also attenuates uptake. Accordingly, we observed reduced FITC-albumin endocytosis following silencing of Rab11 (Fig. 5D-E), suggesting that reduced uptake and intensified degradation partially compensate for compromised recycling. We further observed a diminished FITC-albumin endocytosis (Fig. 5D-E) for Rab7-RNAi suggesting that uptake attenuates upon defective degradation as well. We investigated the role of these Rab proteins specifically for nephrin turnover using the live antibody labeling assay. As described above, in control cells, this assay indicated extensive replacement of nephrin at slit diaphragms after 2 hours (control with heterozygous Myc-nephrin Suppl. Fig. 6A-A"). In contrast, nephrocytes expressing Rab5-RNAi at $18{ }^{\circ} \mathrm{C}$ to attain a milder loss-of-function retained the live labeled antibody after 2 hours at the membrane but also within lines likely corresponding to labyrinthine channels (Fig. 5F-G'). Removal of live labeled Mycnephrin thus dependes entirely on endocytosis. Subsequently, we carried out the live antibody labeling assay in nephrocytes expressing Rab7-RNAi and removal of the Myc-antibody indicated unimpaired endocytic uptake despite Rab7-silencing (Fig. 5H-l'). However, cross sections revealed vesicles containing Myc-antibody (Fig. 5H-|'). Hence, the decelerated degradation facilitated tracking of the antibody's endocytosis. Interestingly, a majority of vesicles were positive for the live 
labeled Myc antibody, but negative the total Myc-Nephrin or nephrin co-staining (Figure 5I-I', Suppl. Fig. 6B-C'). This indicates that the live labeled antibody and Myc-nephrin had dissociated extensively upon entry into endosomes. This implies a functional role for endocytosis by shedding of unwanted molecules from nephrin suggesting that constant endocytosis facilitates self-cleansing of the filtration barrier. Finally, we evaluated the impact of silencing of Rab11, which had a similar impact on nephrin turnover as expression of Rab5-RNAi (Fig. 5J-K, quantitation Fig. 5L, schematic Figure 5M). However, while overall nephrin turnover was similarly reduced, Rab11-RNAi did not cause lateral diffusion of slit diaphragm protein into the labyrinthine channels (compare Fig. 5K-K" to Fig. 5F-F"). This suggests that divergent routes of endocytosis are required for nephrin turnover and prevention of lateral diffusion.

\section{Dynamin-dependent endocytosis and raft-mediated endocytosis play distinct roles in filtration barrier maintenance.}

Rab5 orchestrates endocytic sorting downstream of virtually all entry pathways. Since slit diaphragms form in raft domains, nephrin might travel by clathrin- or raft-mediated endocytosis as suggested by findings in vitro ${ }^{16}$. Recently, a role for clathrin-mediated uptake further was suggested by studies in pericardial nephrocytes ${ }^{33}$. To assess the specific role of these uptake pathways for nephrin trafficking, we first inhibited dynamin-mediated endocytosis. This more canonical route of entry includes clathrinmediated endocytosis. To disrupt dynamin short-term, we employed a temperature-sensitive mutant of the Drosophila dynamin gene, shibirets. This variant remains functional at lower temperature but a temperature shift effectively blocks dynamin-mediated endocytosis in nephrocytes at $30{ }^{\circ} \mathrm{C}^{34}$. Nephrocytes were phenotypically normal in animals kept at $18^{\circ} \mathrm{C}$ (Suppl. Fig. 6D-E'). Blocking dynamin for $2 \mathrm{~h}$ by shifting the animals to $30^{\circ} \mathrm{C}$ resulted in a staining pattern of nephrin and Neph1 that phenocopied Rab5-RNAi showing lateral diffusion (Fig. 6A-B"). This suggested that removal of ectopic nephrin requires a dynamin-dependent route of entry. To obtain acute inhibition of raftmediated endocytosis, we exposed nephrocytes ex vivo to Methyl- $\beta$-Cyclodextrin (Cylodextrin) for 2 hours. This compound depletes the plasma membrane of cholesterol which disperses lipid rafts and thereby prevents raft-mediated endocytosis ${ }^{35}$. However, this short-term treatment had no effect on the staining pattern of slit diaphragm proteins (Fig. 6C-D"), suggesting that removal of ectopic nephrin exclusively relies on dynamin-mediated endocytosis. In contrast, when we studied nephrin turnover by live labeling, we observed effective clearance of the live labeled Myc-antibody from the slit diaphragms for shibirets nephrocytes (Fig. 6E-F"' quantitation Fig. 6I, Neph1 co-staining Suppl. 
Fig. 6F). Hence, slit diaphragm turnover does not require dynamin. However, when we dispersed lipid rafts by Cyclodextrin, we observed a strong reduction in nephrin turnover, using the live antibody labeling assay (Fig. 6G-H”, quantitation Fig. 6I). This indicates that raft-mediated endocytosis is required for the rapid internalization of nephrin residing within the slit diaphragm. We conclude that selective transport routes regulate free nephrin versus slit diaphragm-associated nephrin in vivo.

\section{Flotillin2-mediated endocytosis is required for nephrin turnover in Drosophila nephrocytes}

We next sought to identify the mediator that promotes raft-dependent endocytosis of nephrin. Since caveolins are absent from the Drosophila genome, we hypothesized that flotillins play this role. Flotillins associate with the inner leaflet of the plasma membrane initiating raft-mediated endocytosis in response to phosphorylation by the kinase $\mathrm{Fyn}^{36-38}$. We expressed flotillin2-RNAi in nephrocytes and observed impaired nephrocyte function (Fig. 7A-C). Staining nephrocytes for fly nephrin and Neph1, we observed a localized breakdown of slit diaphrams on sections of the cell surface similar to prolonged Rab5 silencing with incomplete penetrance (Fig. 7D-E'”). Since some animals showed no overt phenotype similar to short-term Cyclodextrin (Suppl. Fig. 7A-B"'), we hypothesized that the localized breakdown of slit diaphragm may only occur as a long-term consequence. Importantly, when we performed the live antibody labeling after silencing flotillin2, we detected strongly diminished nephrin turnover (Fig. 7F-G). This suggests that nephrocytes exercise the specific nephrin turnover by flotillin2-dependent endocytosis. Studying size-dependent permeability of slit diaphragms upon silencing of flo2, we observed a phenocopy of Rab5-RNai with relatively stronger reduction of uptake for the tracer closer to the size cut-off of the nephrocyte filtration barrier that is between $66-80 \mathrm{kDa}^{29}$ (Fig. 7H-J). Another flo2-RNAi recapitulated the observed effects on FITC-albumin uptake, slit diaphragm protein stainings and altered filtration barrier permeability (Suppl. Fig. 7C-J). This confirms that silencing flottilin-mediated turnover is sufficient to block nephrin turnover and alter filtration characteristics. The flo2-dependent nephrin turnover thus appears to be required specifically for cleansing of the nephrocyte filtration barrier to maintain its permeability (working model, Fig. 7K). Taken together, our data indicate how a stable yet dynamic architecture of the filtration barrier facilitates its amazing capabilities and delineate the mechanistic role of endocytosis. Selective routes of vesicular transport are required for maintenance: Canonical dynamin-dependent endocytosis prevents lateral diffusion of slit diaphragm proteins to restrict slit diaphragms to their proper location while flotillin2-dependent endocytosis in lipid rafts facilitates nephrin turnover likely to promote dynamic flexibility but also to cleanse the barrier to prevent clogging during ceaseless filtration. 


\section{DISCUSSION}

Here, we studied the mechanisms of slit diaphragm maintenance and the underlying role of endocytosis in Drosophila nephrocytes. Performing experiments that are currently precluded in mammalian or in vitro models, we combine knock-in lines into the genomic locus of nephrin with live imaging and short-term inhibition of endocytic functions. We observed a stable yet highly dynamic architecture that can be rebuilt after transient disruption. Although nephrin exhibited an extensive half-life exceeding one day, live antibody labeling and FRAP analysis suggested a rapid turnover of nephrin within minutes suggesting rapid cycles of uptake and recycling. To our knowledge, this is the first analysis of slit diaphragms dynamics in vivo. Upon acute silencing of Rab5, which impairs endocytic removal of ectopic nephrin, slit diaphragm proteins diffused laterally into the labyrinthine channels causing eventual breakdown of the architecture. At the same time, the size cut-off of the filtration barrier decreased suggesting incipient filter clogging. Acute disruption of dynamin function and cholesterol depletion revealed that endocytosis is required for two major functions that are attained by selective and independent transport routes: Removal of ectopic nephrin by dynamindependent endocytosis and turnover of nephrin within the slit diaphragm by raft-mediated endocytosis. In this manner, endocytosis restricts and preserves the architecture and cleanses nephrin to preserve permeability of the filtration barrier. We identified flotillin2 as a novel key protein in the raft-mediated turnover of nephrin.

The slit diaphragm is passed by vast amounts of plasma containing a wide range of proteins, metabolites and xenobiotics. Binding of molecules to the slit diaphragm poses a constant threat of filter clogging. It has been a longstanding question how podocytes prevent clogging of the glomerular filter. Our live antibody experiments suggest that nephrin may shed proteins within the lower $\mathrm{pH}$ of endosomes - even antibodies binding with high affinity. We propose that this endosomal cleaning and rapid recycling of nephrin contributes to prevent filter clogging.

The exact speed of turnover is difficult to define. Antibody binding itself might speed up the endocytic turnover in our live antibody labeling assay. However, FRAP analysis equally suggested rapid recovery in an unprovoked setting and similar turnover was described for adherens junctions ${ }^{39}$. While live labeling might overestimate the speed of turnover, the opposite may be true for the FRAP analysis. Directly after photobleaching only bleached nephrin protein is subject to endocytosis while exclusively fluorescent nephrin is delivered by recycling. With progressive observation time, a steady state in the uptake and recycling of bleached and unbleached nephrin may result in a slightly 
premature plateau phase.

The filtration barrier in Drosophila nephrocytes differs anatomically from humans. Nevertheless, the functional and molecular correspondence is striking. The opportunities of genetic manipulation and accessibility for imaging of this podocyte model facilitated unique insights into the fundamental principles of filtration barrier maintenance in vivo. Selective cycles of endocytosis sustain a stable yet flexible filtration barrier. These basic principles are probably conserved in evolution. Since double knock-out mice of Flotillin 1/Flotillin 2 were described without overt renal phenotype ${ }^{40}$, the exact molecular machinery may show partial divergence. It is conceivable that Caveolins may be able to compensate the loss of flotillins in mammals. Future work in higher model organisms will be required to evaluate these principles in the mammalian kidney.

Understanding the mechanistic role of endocytosis will help to identify novel angles for manipulation of the glomerular filtration barrier. Targeting the specific transport processes of nephrin is well-suited to become a promising therapeutic strategy that may be effective across a wide range of glomerular diseases. 


\section{ACKNOWLEDGEMENTS}

We thank C. Meyer for excellent technical support and R. Nitschke, Life Imaging Centre, University of Freiburg, for help with confocal microscopy.

We thank the Developmental Studies Hybridoma Bank (DSHB) for antibodies and the Bloomington Drosophila Stock Center and Vienna Drosophila Resource Center for providing fly stocks.

This research was supported by grants from the Deutsche Forschungsgemeinschaft (DFG) to T.H. (HE 7456/3-1, HE 7456/4-1), and project-ID 431984000 - SFB 1453 (to T. H., M.K. and G.W.), TRR 152 (to M.K.) project-ID 239283807, and Germany's Excellence Strategy: CIBSS - EXC2189 - project-ID 390939984 (to G.W. and M.K.). J.M. and H.H. were supported by the MOTIVATE program of the Medical Faculty of the University of Freiburg. M.C. was supported by the China Scholarship Council. T.H. and K.L. acknowledge support from the Deutsche Gesellschaft für Innere Medizin (DGIM). J.S.P. and A.S. were supported by UNC Kidney Center endowment funds.

\section{AUTHOR CONTRIBUTIONS}

K.L., J.M., H.H., M.C., L.G., D.S., and T.H. performed fly husbandry, crosses, and immunostainings.

K.L. and T.H. performed tracer endocytosis experiments and live antibody labeling assays.

K.L. and J.M. performed live-cell imaging.

M.M. and J.S.P. generated the knock-in of GFP in fly nephrin and performed FRAP experiments T.H. generated the knock-in of Myc in fly nephrin

M.H. and S.K. performed electron microscopy.

G.W. and M.K. contributed to the conceptual presentation of the work in the manuscript.

T.H. conceived of and directed the study. T.H. wrote the manuscript, K.L. prepared the data Figures together with T.H. The manuscript was critically reviewed by all the authors.

\section{DECLARATION OF INTERESTS}

The authors declare no competing interests. 


\section{FIGURE LEGENDS}

Figure 1. Slit diaphragms proteins form a stable architecture that is re-established upon disruption.

(A) Schematic illustrating the nephrocyte ultrastructure and function (surface detail). Molecules destined for removal (shown as green hexagons) pass a bi-layered filtration barrier before being subject to endocytosis within membrane invaginations.

(B) The schematic (upper section) illustrates the slit diaphragm after knock-in of GFP into the nephrin locus. The transmission electron microscopy image (middle section) shows a surface detail of a nephrocyte expressing nephrin-GFP homozygously with regular slit diaphragms. Confocal images (lower section) of a nephrin-GFP nephrocyte show colocalization with endogenous Neph1 (KIRRE) in cross-sectional (upper row) and tangential sections (lower row).

(C-D') Snapshots from a movie obtained by live-cell imaging reveal a stable slit diaphragm pattern in the tangential section (C-C"). This is confirmed by cross-sectional analysis in the same genotype (d-d") where no vesicles for bulk transport of nephrin are observed.

(E-F') Confocal images of tangential section of nephrocytes stained for slit diaphragm proteins while silencing of fly nephrin (sns) is blocked by $G A L 80^{\text {ts }}$ at $18{ }^{\circ} \mathrm{C}$ show a regular staining pattern of slit diaphragm proteins (E-E'). A temperature shift to $31{ }^{\circ} \mathrm{C}$ initiates RNAi expression, resulting in reduction of approximately $50 \%$ of the slit diaphragm protein after two days (F-F").

(G-I') Confocal images of tangential section of nephrocytes that express nephrin (sns)-RNAi and GAL80ts continuously at a non-inhibiting temperature of $31{ }^{\circ} \mathrm{C}$ stained for slit diaphragm proteins nephrin (sns) and Neph1 (Kirre) show an extensive loss of nephrin staining after silencing while a punctate pattern of Neph1 (lacking its binding partner) is observed (G-G'). Both proteins colocalize in short lines indicating renewed formation of slit diaphragms after a temperature shift to $18{ }^{\circ} \mathrm{C}$ that inhibits RNAi expression for three days ( $\left.\mathrm{H}-\mathrm{H}^{\prime \prime}\right)$ Inset in ( $\left.\mathrm{H}^{\prime \prime}\right)$ shows transmission electron microscopy of the same stage with return of sparse and isolated slit diaphragms (red arrow head). The longer lines of slit diaphragm proteins begin to cluster in pairs or triplets after another day, covering a large part of the cell surface in a wide-meshed network (I-I").

(J-J') Slit diaphragm architecture is restored after blocking the expression of nephrin-RNAi for 6 days. 


\section{Figure 2. Live antibody labeling shows rapid nephrin turnover.}

(A) Immunostaining of nephrocyte expressing Myc-nephrin homozygously shows colocalization with endogenous Neph1.

(B) Transmission electron microscopy of a nephrocyte expressing Myc-nephrin homozygously reveals regular slit diaphragms suggesting the tagged protein is functional.

(C) Schematic illustrating live antibody labeling: Living nephrocytes are labeled with anti-Myc antibody (green) that may undergo endocytosis during chasing. Total nephrin stain follows after fixation and permeabilization (red). Colocalization of green and red indicates stable nephrin (surface) or endocytosed nephrin (subcortical). Exclusively green signal indicates antibody dissociation, while new nephrin reaching the surface during the chase period will stain only red.

(D) Confocal microscopy images show cross-sections (top) and tangential sections (bottom) from Myc-nephrin nephrocytes after live antibody labeling without chasing. Extensive colocalization indicates successful nephrin labeling. Nuclei are marked by Hoechst 33342 in blue here and throughout the Figure.

(E) Confocal images analogous to (D) but after one hour of chasing reveal incipient endocytosis.

(F) Confocal images analogous to (D-D") but after two hours of chasing suggest extensive endocytosis. Diffuse intracellular signal from live labeling suggests that internalized antibody separated from nephrin. Exclusively red nephrin signal indicates newly delivered protein.

(G) Quantitation of fluorescence intensity derived from live labeling from conditions in (D-F) expressed as a ratio of surface (slit diaphragm) and subcortical areas confirms significant nephrin turnover ( Mean \pm standard deviation, $n=12-13 P<0.01$ for chase of $1 \mathrm{~h}$ and $\mathrm{P}<0.0001$ for $2 \mathrm{~h}$ ).

(H) Shown are frames from a time lapse movie of nephrin-GFP nephrocytes. The blue box demarcates the region of photobleaching, the yellow box outlines a region of interest where the fluorescence intensity was measured over the length of the FRAP experiment. A loss of fluorescence intensity compared to pre-bleach condition (left panel) is detectable $10 \mathrm{sec}$ after photobleaching (middle panel). After 32 minutes, the fluorescence recovers significantly (right panel).

(I) Quantitative analysis from multiple FRAP experiments ( $n=5$ cells, 8 ROls total, mean \pm standard deviation) reveals an initially rapid recovery of fluorescence intensity that slows to a plateau suggesting a nephrin half-life of $\sim 7$ minutes. The majority of nephrin molecules $(\sim 65 \%)$ are replaced within 30 minutes (mobile fraction). 


\section{Figure 3. Endosomal regulator Rab5 is required for maintenance of slit diaphragms.}

(A) Schematic illustrating endocytic trafficking in a simplified manner shows raft-mediated and clathrin-mediated uptake converging in the early endosome by vesicle fusion. Uptake, early endosome formation and cargo sorting are controlled by Rab5. Sorting may direct cargo either towards degradation, which is promoted by Rab7, or back towards the cell membrane by recycling pathways such as Rab11-dependent recycling.

(B-B') Cross-sectional confocal microscopy images from nephrocytes expressing constitutively active YFP-Rab5 for $24 \mathrm{~h}$ (green) show highly enlarged early endosomes that contain ectopic fly nephrin (magnification see inset). Nuclei are marked by Hoechst 33342 in blue here and throughout the Figure.

(C) Confocal images of nephrocytes with acute silencing of Rab5 for $17 \mathrm{~h}$ reveals brighter sections within the lines of slit diaphragm protein in tangential sections. Lines further are blurry and focally confluent (see also magnified inset).

(D) Cross-sectional images of nephrocytes with short-term silencing of Rab5 show appearance of ectopic slit diaphragm protein below the surface (compare to control Suppl. Fig. 3A-A").

(E-F) Tangential sections (E) and cross sections (F) of nephrocytes with slightly longer silencing of Rab5 for $24 \mathrm{~h}$ stained for nephrin (Sns) and Neph1 (Kirre) reveal progressive thickening of slit diaphragms and localized breakdown of the slit diaphragms in a circumscribed area (white arrow heads).

(G-G'”') Snapshots from a movie obtained by live-cell imaging using confocal microscopy are shown. Nephrocytes expressing nephrin-GFP (heterozygously) are shown after 24 h of acute Rab5 silencing. Increasing gaps and a progressive reduction of slit diaphragms is observed over the course of $1 \mathrm{~h}$.

(H-H') Confocal microscopy images showing cross-sections of nephrocytes after $24 \mathrm{~h}$ of Rab5 silencing are shown. Living cells were exposed to FITC-albumin (green) for 15 min before fixation and staining for nephrin (red). Cells show significant endocytosis of FITC-albumin indicating cell viability and residual endocytic activity despite silencing of Rab5. Ectopic nephrin and FITC-albumin do not colocalize, indicating that ectopic nephrin is not found within a subcellular compartment that is also destination for recently endocytosed cargo. 


\section{Figure 4. Endocytosis prevents lateral diffusion of nephrin and preserves filter permeability.}

(A) Schematic illustrates the assay for visualization of labyrinthine channels. Nephrocytes are fixed briefly before exposure to Texas Red-dextran that enters the channels by passive diffusion.

(B) Confocal microscopy image of a control nephrocyte is stained for Neph1 (green) together with labeling of the channels by Texas-Red-dextran (10 kDa, red). Channels extend directly below the slit diaphragms. Nuclei are marked by Hoechst 33342 in blue here and throughout the Figure.

(C) Confocal images of nephrocytes with short-term silencing of Rab5 show mislocalized fly nephrin below the cell surface that colocalizes significantly with the labyrinthine channels visualized by TexasRed-dextran (10 kDa).

(D-E'") Snapshots from movies obtained by live-cell imaging are shown. Nephrocytes express nephrin-GFP (heterozygously) concomitant with Rab5-RNAi for $24 \mathrm{~h}$. Fusion and cluster formation (white arrow heads in panels C) of fly nephrin precedes appearance of gaps (D-D'”). Similarly, formation of protrusions of slit diaphragm proteins from the cell surface is followed by a formation of vesicles (E-E'”, white arrowheads).

(F) Electron microscopy (EM) image from a cross section through the surface of a control nephrocyte reveals regular slit diaphragms bridging the membrane invaginations called labyrinthine channels.

(G) EM image from a section through the surface of a nephrocyte expressing Rab5-RNAi acutely for $24 \mathrm{~h}$ demonstrates ectopic formation of slit diaphragms forming rosette-like structures within the labyrinthine channels (red circles, magnification on the right).

(H-J') Confocal microscopy images of nephrocytes after simultaneous uptake of tracers FITCalbumin (66 kDa, green) and Texas-Red-dextran (10 kDa) are shown. Control nephrocytes show robust uptake of both tracers $\left(\mathrm{H}-\mathrm{H}^{\prime \prime}\right)$. Silencing of Rab5 acutely for $24 \mathrm{~h}$ shows a stronger decrease in the uptake of the larger tracer FITC-albumin compared to smaller Texas-Red-dextran (H-H"). Both tracers are equally reduced upon nephrin silencing (I-I').

(K) Quantitation of fluorescence intensity expressed as a ratio of Texas-Red-Dextran/FITC-albumin (small/large tracer) confirms a disproportionate reduction for the larger tracer for Rab5-RNAi but not nephrin-RNAi (mean \pm standard deviation, $\mathrm{n}=9$, $\mathrm{P}<0.0001$ for Rab5-RNAi, $\mathrm{P}>0.05$ for nephrin-RNAi). (L) Schematic illustrates how incipient filter clogging affects uptake of larger tracer disproportionately. 
Figure 5. Endocytic uptake and Rab11-dependent recycling are required for slit diaphragm maintenance.

(A-A') Stainings of Rab7-RNAi nephrocytes reveal an additional faint signal for nephrin but not for Neph1 that likely reflects accumulation of nephrin upon defective degradation. Tangential sections (insets) show a regular fingerprint-like pattern, indicating undisturbed slit diaphragm formation. Nuclei are marked by Hoechst 33342 in blue here and throughout the Figure.

(B) EM image of Rab7-RNAi nephrocyte shows normal slit diaphragms and large vesicles.

(C) EM of nephrocyte expressing Rab11-RNAi reveals reduction of labyrinthine channels with multiple slits close to the cell surface (see inset) and expansion of lysosomes (red asterisks).

(D) FITC-albumin endocytosis as assay for nephrocyte function shows reduced uptake for Rab7RNAi (lower panels) and Rab11-RNAi (upper panels) using Dorothy-GAL4 or prospero-GAL4 compared to the respective controls.

(E) Quantitation of results from (D) in ratio to a control experiment performed in parallel (mean \pm standard deviation, $\mathrm{n}=11-14$ per genotype, $\mathrm{P}<0.0001$ for Rab7-RNAi and $\mathrm{n}=9$ per genotype $\mathrm{P}<0.0001$ for Rab11-RNAi). Sidak post hoc analysis was used to correct for multiple comparisons.

(F-K') Confocal microscopy images of tangential sections (F-F", H-H", J-J") and cross-sections (GG", I-I", K-K") of Myc-nephrin nephrocytes after live antibody labeling and 2 hours of chasing are shown for the indicated genotypes. Silencing of $R a b 5$ at $18{ }^{\circ} \mathrm{C}$ was obtained before flies were adapted to $25^{\circ} \mathrm{C}$ for $1 \mathrm{~h}$ (F-G”). Live labeling (green) and total stain (red) show near-complete colocalization for Rab5-RNAi (F-G'), indicating disrupted nephrin turnover. Extensive amounts of subcortical nephrin are revealed in cross sections (G-G'), compatible with lateral diffusion into the membrane invaginations. Cells expressing Rab7-RNAi after live antibody labeling show undisturbed nephrin turnover as the live labeled antibody is removed from the surface ( $\left.\mathrm{H}-\mathrm{H}^{\prime \prime}\right)$. Cross sections of Rab7RNAi nephrocytes reveal numerous subcortical vesicles that partially show isolated signal for the live labeling, indicating the antibody disengaged from nephrin (I-l'). Nephrocytes expressing Rab11-RNAi show strong retention of live labeled nephrin on the cell surface (J-J'), suggesting impaired turnover. Cross sections show the antibody on the surface, but not in labyrinthine channels (K-K').

(G) Quantitation of results from (F-K') expressed as ratio of the fluorescence intensity between surface and subcortical region for individual cells (mean \pm standard deviation, $n=11-13$ per genotype, $\mathrm{P}<0.0001$ for Rab5-RNAi, $\mathrm{P}>0.05$ for Rab7-RNAi and $\mathrm{P}<0.0001$ for Rab11-RNAi).

(H) Schematic illustrates findings studying nephrin live labeling upon silencing of Rab5/Rab7/Rab11. 
Figure 6. Differential transport through dynamin-mediated or raft-mediated endocytosis is required for slit diaphragm maintenance in nephrocytes.

(A-B') Confocal image of nephrocyte stained for slit diaphragm proteins carrying a temperaturesensitive variant (G141S) of shibire, the Drosophila dynamin, homozygously. The mutant protein is functional at lower temperatures but lacks function at $30^{\circ} \mathrm{C}$ and the animals were exposed to $30{ }^{\circ} \mathrm{C}$ for $2 \mathrm{~h}$ before staining. Cross sections show accumulation of subcortical slit diaphragm protein in clusters and short lines protruding from the surface (A-A"). Tangential sections indicate a mild confluence and few brighter clusters of slit diaphragm proteins (B-B").

(C-D') Confocal images of control nephrocytes treated with Cylodextrin for $2 \mathrm{~h}$ ex vivo to inhibit raftmediated endocytosis, show a regular staining pattern of slit diaphragm proteins in cross-sectional (C-C") and tangential planes (D-D").

(E-H') Confocal microscopy images showing tangential sections (panels $E$ and $G$ ) and crosssections (panels $F$ and $G$ ) of nephrocytes carrying one copy of the genomic Myc-nephrin after live antibody labeling with 2 hours of chase period are for the indicated genotypes or interventions. Shibirets animals show intense nephrocyte turnover in the live labeling assay despite exposure to a temperature of $31^{\circ} \mathrm{C}$ for $2 \mathrm{~h}$ which inhibits function of the fly dynamin during that period (E-F"). In contrast, blocking raft-mediated endocytosis for $2 \mathrm{~h}$ by Cyclodextrin in control nephrocytes strongly diminishes nephrin turnover and a large amount of the live-labeled antibody is retained (G-H'). This suggests that nephrin turnover depends on raft-mediated endocytosis that occurs independent from dynamin function.

(I) Quantitation of results from (E-H") expressed as ratio of the fluorescence intensity between surface and subcortical region for individual cells (mean \pm standard deviation, $n=11-12$ per genotype, $\mathrm{P}>0.05$ for shibire ${ }^{t s}$, and $\mathrm{P}<0.0001$ for Cyclodextrin treatment). 


\section{Figure 7. Flotillin2-mediated endocytosis is required for nephrin turnover in Drosophila nephrocytes.}

(A-B) Confocal microscopy images of nephrocytes after uptake of FITC-albumin as read-out of nephrocyte function are shown. Control nephrocytes exhibit stronger uptake $(A)$ than nephrocytes expressing flo2-RNAi (B).

(C) Quantitation of results analogous to $(A-B)$ in ratio to a control experiment performed in parallel (mean \pm standard deviation, $\mathrm{n}=7$ per genotype, $\mathrm{P}<0.0001$ for flo2-RNAi).

(D-E') Confocal images of nephrocytes expressing flo2-RNAi show localized breakdown of slit diaphragms in cross-sectional (D-D') and tangential planes (E-E').

(F-F') Confocal microscopy images in tangential sections (upper row) and cross-sections (lower row) of nephrocytes are shown after live antibody labeling with 2 hours of chasing. Animals express flo2RNAi under control of Dorothy-GAL4. Nephrin turnover is strongly reduced compared to control (Suppl. Fig. 6A).

(G) Quantitation of results from (F) compared to control experiments. Results are expressed as ratio of the fluorescence intensity between surface and subcortical regions for individual cells (mean \pm standard deviation, $\mathrm{n}=11$ per genotype, $\mathrm{P}<0.0001$ for flo2-RNAi).

(H-I') Confocal microscopy images of nephrocytes after simultaneous uptake of FITC-albumin (66 kDa, green) and Texas-Red-dextran (10 kDa) are shown. Control nephrocytes show significant uptake of both tracers ( $\left.\mathrm{H}-\mathrm{H}^{\prime \prime}\right)$. Silencing of flo2 causes a stronger decrease in the uptake of the larger tracer FITC-albumin compared to smaller Texas-Red-dextran (I-l').

(J) Quantitation of fluorescence intensity expressed as a ratio of Texas-Red-Dextran/ FITC-albumin (small/large tracer) confirms a disproportionate reduction for flo2-RNAi (mean \pm standard deviation, $\mathrm{n}=9 \mathrm{P}<0.001$ for flo2-RNAi).

(K) Schematic illustrating the proposed mechanistic role of endocytosis for maintenance of the filtration barrier. Left: Ectopic fly nephrin within the channels is removed by clathrin-dependent endocytosis that returns most of the protein to the surface through recycling pathways. The nephrin that is bound within the slit diaphragm complex is subject to turnover in a shorter circuit that is raftmediated and feeds into recycling as well. Right: Upon disruption of endocytosis filtration is impaired by clogging of the filter due to lack of cleansing and the architecture of the slit diaphragms is disturbed by unhindered lateral diffusion of slit diaphragm protein. 


\section{METHODS}

\section{Fly strains and husbandry.}

Flies were reared on standard food at room temperature, $18{ }^{\circ} \mathrm{C}, 25^{\circ} \mathrm{C}$ or $31{ }^{\circ} \mathrm{C}$ as indicated. Overexpression and transgenic RNAi studies were performed using the UAS/GAL4 system (RNAi crosses grown at $25^{\circ} \mathrm{C}$ or $31^{\circ} \mathrm{C}$ ). Nephrocyte indicates the subtype of garland cell nephrocytes throughout the manuscript. Stocks obtained from the Bloomington Drosophila Stock Center were UAS-nephrin (sns)-RNAi (\#64872), UAS-Rab5-RNAi (34832\#), UAS-Rab5 ${ }^{\text {S43N }}$ (dominant negative) (\#42704), UAS-YFP-Rab5 ${ }^{\text {Q88L }}$ (constitutively active, \#9774), UAS-Rab7-RNAi (\#27051), UAS-YFPRab7 ${ }^{\text {T22N }}$ (dominant negative \#9778), UAS-Rab11-RNAi (\#42709), UAS-flo2-RNAi (\#40833), and

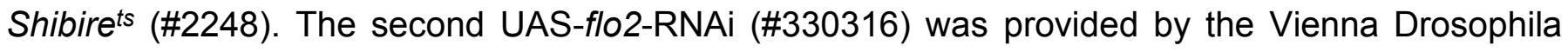
RNAi Center (VDRC), prospero-GAL431 and Dorothy-GAL4 (\#6903; BDSC) were used with or without tub-GAL80ts (\#7018; BDSC) to control expression in nephrocytes. UAS-GFP-RNAi (\#41553; BDSC) or wild type ( $\left.\mathrm{yw}^{1118}\right)$ were crossed to GAL4-drivers as control.

\section{Generation of nephrin-GFP.}

Nephrin-GFP was generated by using CRISPR/piggyBac to introduce a C-terminal super folder GFP at the fly nephrin (sns) locus using the scarless gene editing approach ${ }^{41}$. A single guide RNA (gRNA) targeting the 3' end of sns was cloned into pU6-Bbsl-chiRNA. A dsDNA donor template for homologydirected repair with $1 \mathrm{~kb}$ homologies upstream and downstream was generated by PCR amplification from genomic DNA and assembly into pScarlessHD-sfGFP-DsRed by Gibson DNA Assembly (New England Biolabs). A mixture of both plasmids was injected into flies expressing Cas 9 under nos regulatory sequences by BestGene. CRISPR edited lines were identified by the presence of DsRed eye fluorescence. We removed PBac-3xP3-DsRed-PBac sequences in these stocks by precise excision of the PBac transposable element by crossing to tub-Pbac flies (\#8283; BDSC) and established the resulting nephrin-GFP as homozygous stocks.

\section{Live imaging using nephrin-GFP.}

Nephrin-GFP expressing nephrocytes were dissected in phosphate buffered saline (PBS) immediately before mounting on slides with cover slips in Schneider's medium containing $1 \%$ low melting agarose (Carl Roth $\mathrm{GmbH}$ ). The slide was put on an ice block for a few seconds and then left on room temperature for 5 minutes to allow the agarose solution to become solid. Imaging was performed using a Zeiss LSM 880 laser scanning microscope employing electronic autofocus over the course of up to $1 \mathrm{~h}$. 


\section{Generation of genomic Myc-nephrin.}

For generation of genomic Myc-nephrin, we targeted the second exon of sns using using pCFD3 (\#49410; Addgene, target sequence: AGTGCCAGGTGGGACCGGCT). A homology-directed repair template was assembled by a step-wise amplification of homologies upstream and downstream of the second exon of fly nephrin (sns) using a vector from the BACPAC library that covered the sns locus. A Myc sequence was inserted directly adjacent to the target's (mutated) PAM. DsRed cDNA under P3 promoter flanked by loxP sites was derived from pHD-DsRed (Addgene plasmid \#51434) and placed into the flanking intron that preceded the downstream homology. Twelve synonymous changes were introduced between Myc and the exon boundary to avoid alignment in the interjacent section. A mixture of both plasmids was injected into flies expressing Cas 9 under nos regulatory sequences (\#54591; BDSC) by BestGene. CRISPR edited lines were identified by the presence of DsRed eye fluorescence and the DsRed marker was removed by crossing to flies expressing cre recombinase (\#1092; BDSC). We established the resulting Myc-nephrin flies as a homozygous stock.

\section{Fluorescent tracer uptake.}

Fluorescent tracer uptake in nephrocytes to evaluate nephrocyte function was performed as previously described ${ }^{29}$. Briefly, nephrocytes were dissected in PBS and incubated with FITC-albumin (Sigma) for 30 seconds. After a fixation step of $5 \mathrm{~min}$ in $8 \%$ paraformaldehyde cells were rinsed in PBS and exposed to Hoechst 33342 (1:1000) for 20 seconds and mounted in Roti-Mount (Carl Roth). Cells were imaged using a Zeiss LSM 880 laser scanning microscope. Quantitation of fluorescent tracer uptake was performed with ImageJ software. The results are expressed as a ratio to a control experiment with flies carrying the (heterozygous) GAL4 transgene but no UAS that was performed in parallel.

The parallel recording of two fluorescent tracers of different size to study the passage of the slit diaphragm was carried out in the same way as the assay for nephrocyte function, except that nephrocytes were simultaneously incubated with tracers FITC-albumin $(0.2 \mathrm{mg} / \mathrm{ml})$ and Texas-RedDextran (10 kDa, $0.2 \mathrm{mg} / \mathrm{ml}$ ) for 30 seconds after dissection. Cells were imaged using a Zeiss LSM 880 laser scanning microscope. Image quantitation was performed with ImageJ software for each channel separately.

\section{Immunofluorescence studies and TUNEL detection using Drosophila tissue.}

For immunofluorescence, nephrocytes were dissected, fixed for 20 minutes in PBS containing 4\% paraformaldehyde, and stained according to the standard procedure. The following primary 
antibodies were used: rabbit anti-sns ${ }^{42}$ (1:200, gift from S. Abmayr) and guinea pig anti-Kirre ${ }^{43}$ (1:200, gift from S. Abmayr). Other antibodies used were rabbit anti-Rab5 (ab18211, abcam), mouse antiRab7 (Rab7, DSHB), mouse anti-Myc (9E10; DSHB), mouse anti- c-Myc (sc-40; Santa Cruz Biotechnology), and rabbit anti-RAB11 (\#5589S; Cell Signaling Technology). Apoptotic cells were visualized using the In Situ Cell Death Detection Kit (Sigma/Roche) according to the manufacturer's instructions. For imaging, a Zeiss LSM 880 laser scanning microscope was used. Image processing was done by ImageJ and GIMP software.

\section{Live antibody labeling and internalization}

For live antibody labeling, we modified previously published protocols ${ }^{44,45}$. Nephrocytes were dissected in PBS and immediately incubated with primary antibody (mouse anti- c-Myc, see above, 1:100) for $30 \mathrm{~min}$ at $4{ }^{\circ} \mathrm{C}$ before rinsing several times with cold PBS to remove unbound antibody. The living cells labeled with antibody were chased at $29^{\circ} \mathrm{C}$ for the indicated time. Then, the tissue was fixed, permeabilized and washed before Alexa488-coupled anti-mouse secondary antibody was applied. To obtain total nephrin staining after this step, incubation with anti-Myc primary antibody was repeated overnight. After the preceding permeabilization, the entire nephrin protein of the cell was now accessible to the anti-Myc antibody in this step. Finally, for detection of total nephrin staining an Alexa-568-coupled anti-mouse secondary was employed. For imaging, a Zeiss LSM 880 laser scanning microscope was utilized. Image processing was done by ImageJ and GIMP software.

\section{FRAP analysis}

Nephrocytes from wandering 3rd instar expressing nephrin-GFP were dissected and mounted in Schneider's media. FRAP experiments were conducted on a Zeiss 880 confocal microscope. Prebleach images (2-4 frames) were first acquired, followed by a single photobleaching event consisting of $30-40$ scans of the $488 \mathrm{~nm}$ laser at $100 \%$ power. Photobleaching was confined to a region of interest (ROI) covering a small region of slit diaphragms, as indicated by enriched nephrin-GFP at the cell surface. After bleaching, standard time series acquisition (images acquired every 10-15 seconds) continued for the remainder of the movie. To counter any sample drift, manual correction of the z-axis was performed throughout the time series acquisition. An ROI was drawn over the slit diaphragm-containing edge of the cell within the photobleached area using ImageJ. We measured mean gray value in the ROI at each time point, and subtracted the background from an adjacent extracellular region outside the ROI. For each cell, we measured 1-2 ROIs. We calculated the mean of the mean gray values for the pre-bleach period, and then standardized all subsequent measures 
of signal intensity by expressing them as a percentage of the pre-bleach signal. We then averaged the percent mean gray values for all acquisition time points within each minute of the post-bleach time lapse series. We combined these data from all the FRAP experiments, treating each ROI from each cell as a replicate, to determine the mean and SEM at each time point.

\section{Channel diffusion assay}

To visualize the nephrocytes' membrane invaginations we dissected nephrocytes and fixed them briefly for $5 \mathrm{~min}$ in PBS containing 4\% paraformaldehyde. Shorter fixation preserves slit diaphragm permeability. Cells were then incubated for $10 \mathrm{~min}$ in FITC-albumin (Sigma) or Texas-Red-dextran (10 kD; Thermofisher) to allow tracer diffusion into the channels. Our regular staining protocol was completed according to standard procedure after a second fixation step in paraformaldehyde for 15 $\min$.

\section{Electron microscopy.}

For transmission electron microscopy (TEM) nephrocytes were dissected and fixed in $4 \%$ formaldehyde and $0.5 \%$ glutaraldehyde in $0.1 \mathrm{M}$ cacodylate buffer, $\mathrm{pH} 7.4$. TEM was carried out using standard techniques.

\section{Statistics.}

Paired t test was used to determine the statistical significance between two interventions. Oneway ANOVA followed by Dunnett's correction for multiple testing (unless otherwise indicated) was used for multiple comparisons (GraphPad Prism software). Measurements were from distinct samples. Asterisks indicate significance as follows: ${ }^{*} P<0.05,{ }^{* *} P<0.01,{ }^{* * *} P<0.001,{ }^{* * *} P<0.0001$. A statistically significant difference was defined as $P<0.05$. Error bars indicate standard deviation (SD). 


\section{TABLE OF SUPPL. CONTENT}

Suppl. Fig. 1: Validation of nephrin-GFP and additional time points for disruption and reassembly of slit diaphragms.

Suppl. Fig. 2: Validation of Myc-nephrin

Suppl. Fig. 3: Validation and control experiments for loss-of-function of Rab5.

Suppl. Fig. 4: Channel assay reveals loss of invaginations upon silencing of nephrin and impaired slit diaphragm passage upon silencing of Rab5.

Suppl. Fig. 5: Validation and controls for Rab7 and Rab11.

Suppl. Fig. 6: Additional images for live antibody labeling and validation and controls for Shibirets.

Suppl. Fig. 7: Silencing flotillin2 using a second RNAi line confirms reduced FITC-albumin uptake and altered permeability of the filtration barrier.

Suppl. Video 1: Nephrin-GFP in larval nephrocyte tangential section

Suppl. Video 2: Nephrin-GFP in larval nephrocyte cross section.

Suppl. Video 3: Nephrin-GFP in larval nephrocyte upon transient nephrin silencing.

Suppl. Video 4: Nephrin-GFP in larval nephrocyte upon acute Rab5 silencing (surface).

Suppl. Video 5: Nephrin-GFP in larval nephrocyte upon acute Rab5 silencing (breakdown of slit diaphragms).

Suppl. Video 6: Nephrin-GFP in larval nephrocyte upon acute Rab5 silencing (lateral diffusion). 


\section{References}

1. Scott, R.P. \& Quaggin, S.E. Review series: The cell biology of renal filtration. J Cell Biol 209, 199-210 (2015).

2. Butt, L., et al. A molecular mechanism explaining albuminuria in kidney disease. Nature metabolism 2, 461474 (2020).

3. Kestila, M., et al. Positionally cloned gene for a novel glomerular protein--nephrin--is mutated in congenital nephrotic syndrome. Mol Cell 1, 575-582 (1998).

4. Holzman, L.B., et al. Nephrin localizes to the slit pore of the glomerular epithelial cell. Kidney Int 56, 14811491 (1999).

5. Donoviel, D.B., et al. Proteinuria and perinatal lethality in mice lacking NEPH1, a novel protein with homology to NEPHRIN. Mol Cell Biol 21, 4829-4836 (2001).

6. Gerke, P., Huber, T.B., Sellin, L., Benzing, T. \& Walz, G. Homodimerization and heterodimerization of the glomerular podocyte proteins nephrin and NEPH1. J Am Soc Nephrol 14, 918-926 (2003).

7. Barletta, G.M., Kovari, I.A., Verma, R.K., Kerjaschki, D. \& Holzman, L.B. Nephrin and Neph1 co-localize at the podocyte foot process intercellular junction and form cis hetero-oligomers. J Biol Chem 278, 19266 19271 (2003).

8. Grahammer, F., Schell, C. \& Huber, T.B. The podocyte slit diaphragm--from a thin grey line to a complex signalling hub. Nature reviews. Nephrology 9, 587-598 (2013).

9. Boute, N., et al. NPHS2, encoding the glomerular protein podocin, is mutated in autosomal recessive steroid-resistant nephrotic syndrome. Nat Genet 24, 349-354 (2000).

10. Winn, M.P., et al. A mutation in the TRPC6 cation channel causes familial focal segmental glomerulosclerosis. Science 308, 1801-1804 (2005).

11. Reiser, J., et al. TRPC6 is a glomerular slit diaphragm-associated channel required for normal renal function. Nat Genet 37, 739-744 (2005).

12. Martin, C.E. \& Jones, N. Nephrin Signaling in the Podocyte: An Updated View of Signal Regulation at the Slit Diaphragm and Beyond. Front Endocrinol (Lausanne) 9, 302 (2018).

13. Grahammer, F., et al. A flexible, multilayered protein scaffold maintains the slit in between glomerular podocytes. JCI Insight 1(2016).

14. Inoue, K. \& Ishibe, S. Podocyte endocytosis in the regulation of the glomerular filtration barrier. Am J Physiol Renal Physiol 309, F398-405 (2015).

15. Quack, I., et al. beta-Arrestin2 mediates nephrin endocytosis and impairs slit diaphragm integrity. Proc Natl Acad Sci U S A 103, 14110-14115 (2006).

16. Qin, X.S., et al. Phosphorylation of nephrin triggers its internalization by raft-mediated endocytosis. $J A m$ Soc Nephrol 20, 2534-2545 (2009).

17. Harris, D.P., et al. Requirement for class II phosphoinositide 3-kinase C2alpha in maintenance of glomerular structure and function. Mol Cell Biol 31, 63-80 (2011).

18. Bechtel, W., et al. Vps34 deficiency reveals the importance of endocytosis for podocyte homeostasis. $J$ Am Soc Nephrol 24, 727-743 (2013).

19. Soda, K., et al. Role of dynamin, synaptojanin, and endophilin in podocyte foot processes. J Clin Invest 122, 4401-4411 (2012).

20. Tossidou, I., et al. Podocytic PKC-alpha is regulated in murine and human diabetes and mediates nephrin endocytosis. PLoS One 5, e10185 (2010).

21. Teng, B., et al. CIN85 Deficiency Prevents Nephrin Endocytosis and Proteinuria in Diabetes. Diabetes 65, 3667-3679 (2016). 
22. Quack, I., et al. PKC alpha mediates beta-arrestin2-dependent nephrin endocytosis in hyperglycemia. J Biol Chem 286, 12959-12970 (2011).

23. Konigshausen, E., et al. Angiotensin II increases glomerular permeability by beta-arrestin mediated nephrin endocytosis. Sci Rep 6, 39513 (2016).

24. Hermle, T., et al. GAPVD1 and ANKFY1 Mutations Implicate RAB5 Regulation in Nephrotic Syndrome. J Am Soc Nephrol 29, 2123-2138 (2018).

25. Dorval, G., et al. TBC1D8B Loss-of-Function Mutations Lead to X-Linked Nephrotic Syndrome via Defective Trafficking Pathways. Am J Hum Genet 104, 348-355 (2019).

26. Kampf, L.L., et al. TBC1D8B Mutations Implicate RAB11-Dependent Vesicular Trafficking in the Pathogenesis of Nephrotic Syndrome. J Am Soc Nephrol 30, 2338-2353 (2019).

27. Denholm, B. \& Skaer, H. Bringing together components of the fly renal system. Curr Opin Genet Dev 19, 526-532 (2009).

28. Zhuang, S., et al. Sns and Kirre, the Drosophila orthologs of Nephrin and Neph1, direct adhesion, fusion and formation of a slit diaphragm-like structure in insect nephrocytes. Development 136, 2335-2344 (2009).

29. Hermle, T., Braun, D.A., Helmstadter, M., Huber, T.B. \& Hildebrandt, F. Modeling Monogenic Human Nephrotic Syndrome in the Drosophila Garland Cell Nephrocyte. J Am Soc Nephrol 28, 1521-1533 (2017).

30. Helmstadter, M., et al. Functional study of mammalian Neph proteins in Drosophila melanogaster. PLoS One 7, e40300 (2012).

31. Weavers, H., et al. The insect nephrocyte is a podocyte-like cell with a filtration slit diaphragm. Nature 457, 322-326 (2009).

32. Hochapfel, F., et al. Distinct functions of Crumbs regulating slit diaphragms and endocytosis in Drosophila nephrocytes. Cell Mol Life Sci (2017).

33. Wang, L., Wen, P., van de Leemput, J., Zhao, Z. \& Han, Z. Slit diaphragm maintenance requires dynamic clathrin-mediated endocytosis facilitated by AP-2, Lap, Aux and Hsc70-4 in nephrocytes. Cell \& bioscience 11, 83 (2021).

34. Kosaka, T. \& Ikeda, K. Reversible blockage of membrane retrieval and endocytosis in the garland cell of the temperature-sensitive mutant of Drosophila melanogaster, shibirets1. J Cell Biol 97, 499-507 (1983).

35. Zidovetzki, R. \& Levitan, I. Use of cyclodextrins to manipulate plasma membrane cholesterol content: evidence, misconceptions and control strategies. Biochimica et biophysica acta 1768, 1311-1324 (2007).

36. Glebov, O.O., Bright, N.A. \& Nichols, B.J. Flotillin-1 defines a clathrin-independent endocytic pathway in mammalian cells. Nat Cell Biol 8, 46-54 (2006).

37. Meister, M. \& Tikkanen, R. Endocytic trafficking of membrane-bound cargo: a flotillin point of view. Membranes 4, 356-371 (2014).

38. Otto, G.P. \& Nichols, B.J. The roles of flotillin microdomains--endocytosis and beyond. J Cell Sci 124, 39333940 (2011).

39. de Beco, S., Gueudry, C., Amblard, F. \& Coscoy, S. Endocytosis is required for E-cadherin redistribution at mature adherens junctions. Proc Natl Acad Sci U S A 106, 7010-7015 (2009).

40. Bitsikas, V., Riento, K., Howe, J.D., Barry, N.P. \& Nichols, B.J. The role of flotillins in regulating abeta production, investigated using flotillin 1-/-, flotillin 2-/- double knockout mice. PLoS One 9, e85217 (2014).

41. Bruckner, J.J., et al. Fife organizes synaptic vesicles and calcium channels for high-probability neurotransmitter release. J Cell Biol 216, 231-246 (2017).

42. Bour, B.A., Chakravarti, M., West, J.M. \& Abmayr, S.M. Drosophila SNS, a member of the immunoglobulin superfamily that is essential for myoblast fusion. Genes \& development 14, 1498-1511 (2000).

43. Galletta, B.J., Chakravarti, M., Banerjee, R. \& Abmayr, S.M. SNS: Adhesive properties, localization requirements and ectodomain dependence in $\mathrm{S} 2$ cells and embryonic myoblasts. Mechanisms of development 121, 1455-1468 (2004).

44. Strutt, H., Warrington, S.J. \& Strutt, D. Dynamics of core planar polarity protein turnover and stable assembly into discrete membrane subdomains. Developmental cell 20, 511-525 (2011). 
bioRxiv preprint doi: https://doi.org/10.1101/2022.03.01.482547; this version posted March 2, 2022. The copyright holder for this preprint (which was not certified by peer review) is the author/funder. All rights reserved. No reuse allowed without permission.

45. Hermle, T., Guida, M.C., Beck, S., Helmstadter, S. \& Simons, M. Drosophila ATP6AP2/VhaPRR functions both as a novel planar cell polarity core protein and a regulator of endosomal trafficking. EMBO J 32, 245259 (2013). 
bioRxiv preprint doi: https://doi.org/10.1101/2022.03.01.482547; this version posted March 2, 2022. The copyright holder for this preprint

(which was not certified by peer review) is the author/funder. All rights reserved. No reuse allowed without permission.

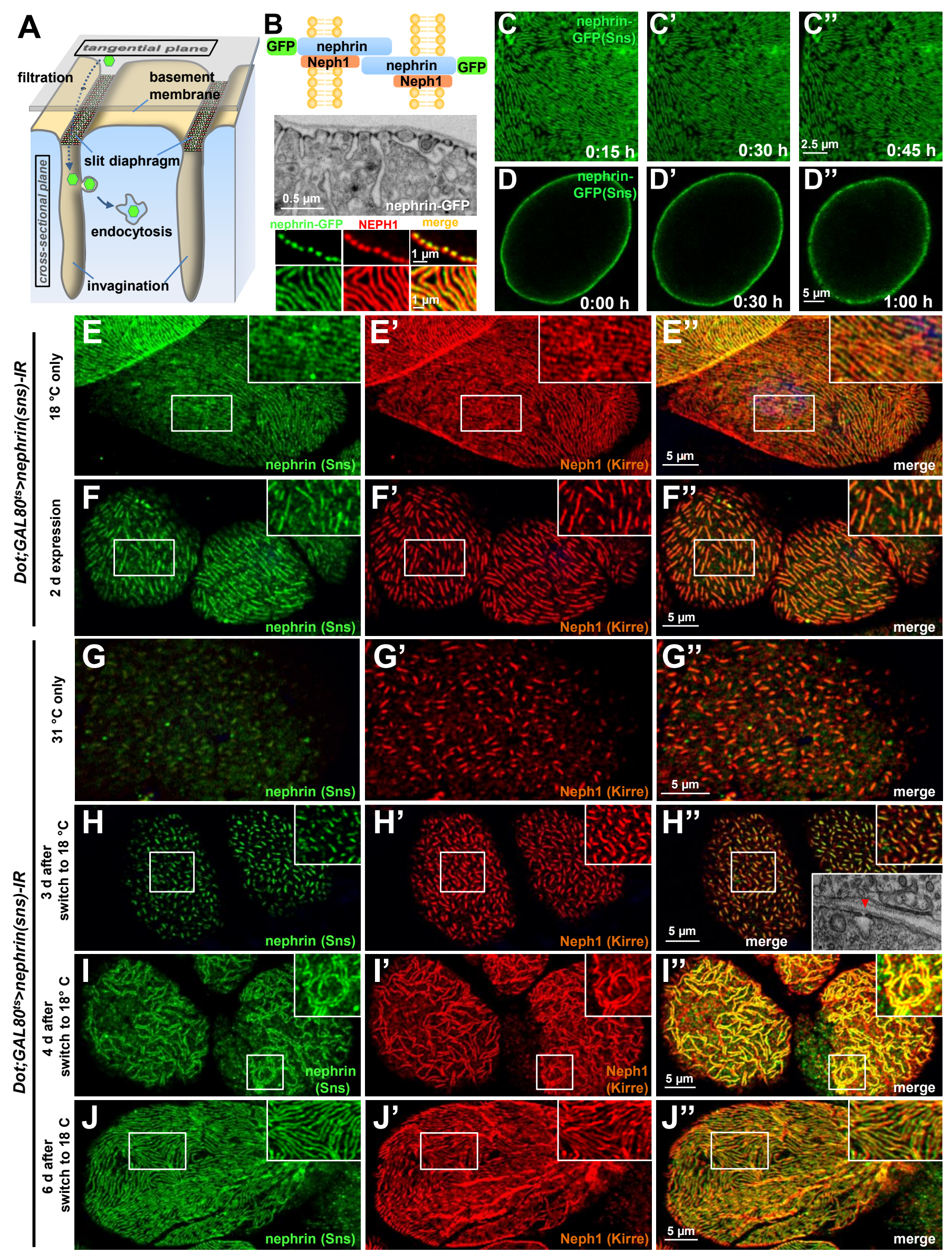

Fig. 1 
bioRxiv preprint doi: https://doi.org/10.1101/2022.03.01.482547; this version posted March 2, 2022. The copyright holder for this preprint (which was not certified by peer review) is the author/funder. All rights reserved. No reuse allowed without permission.

A

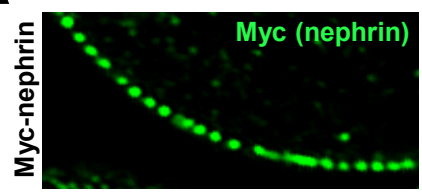

C
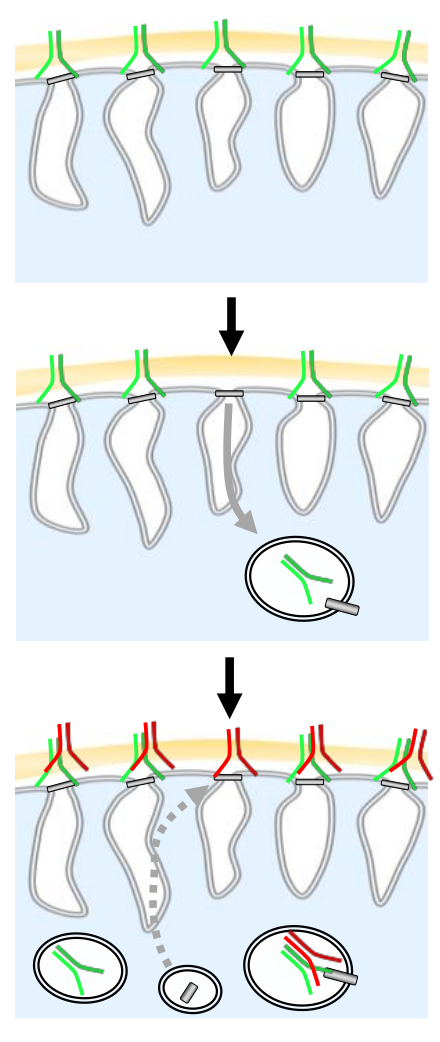

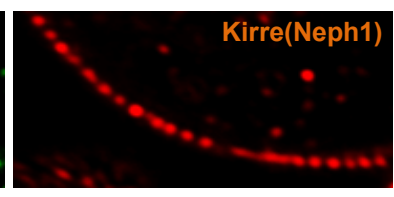

Myc-nephrin

(live labeling 25 min)
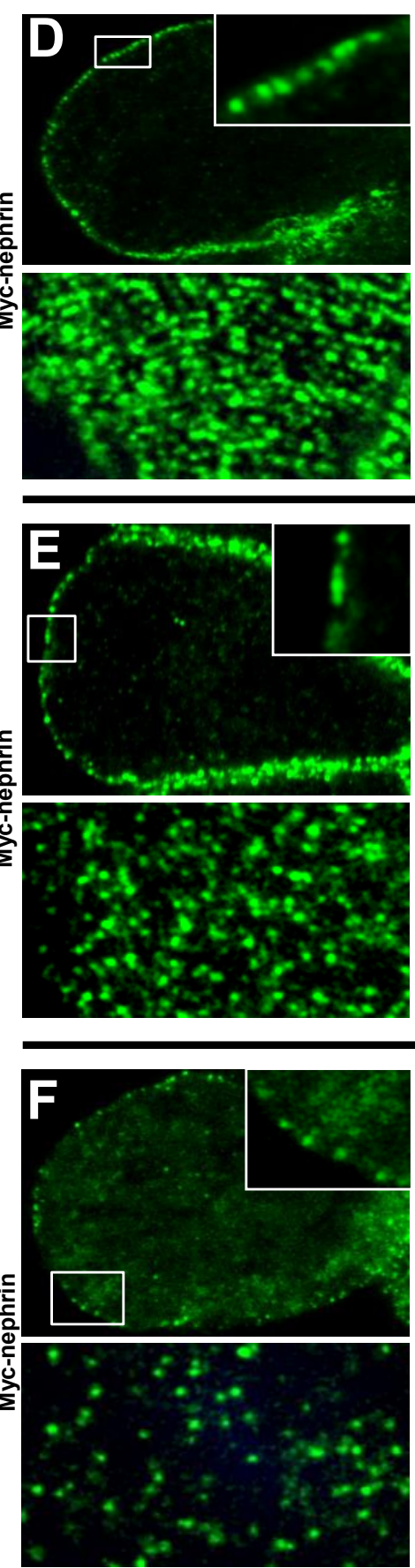

oh chase 1 h chase 2 h chase

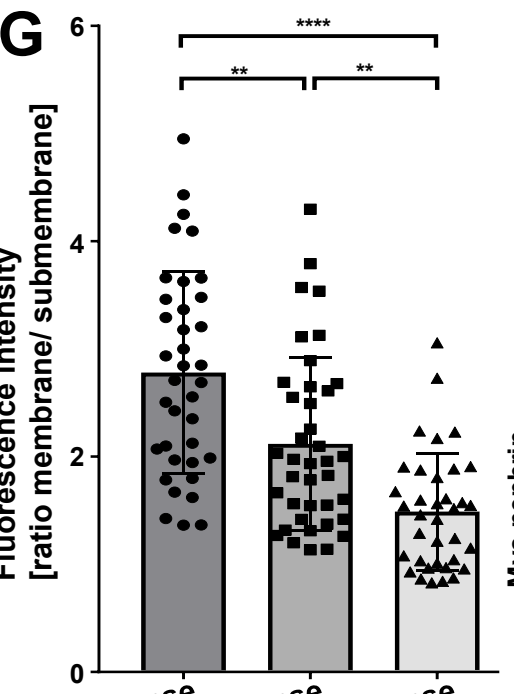

H
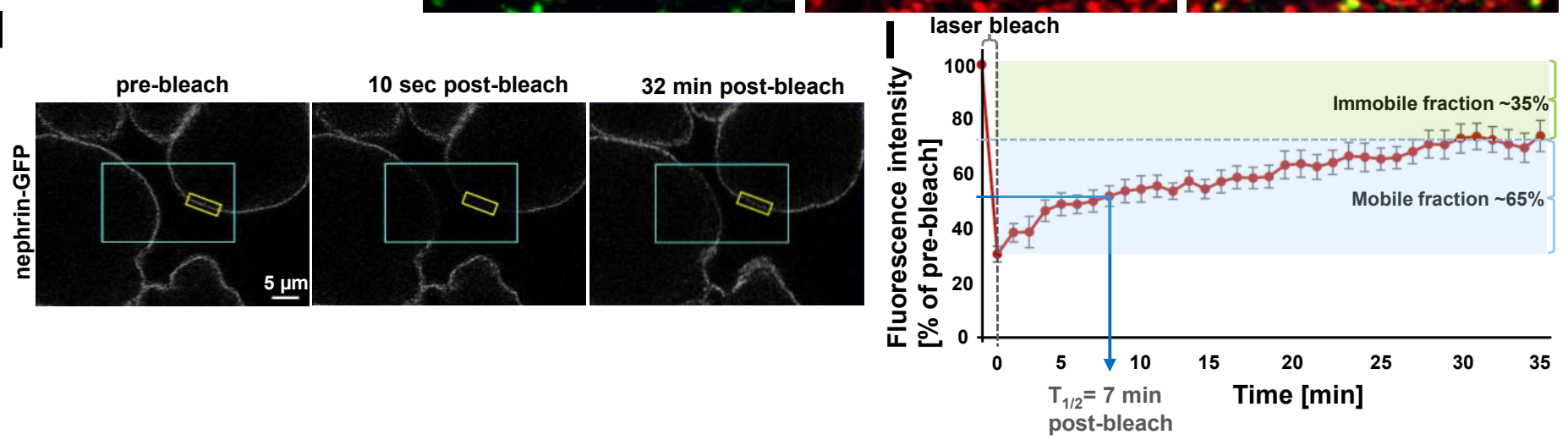

Fig. 2 


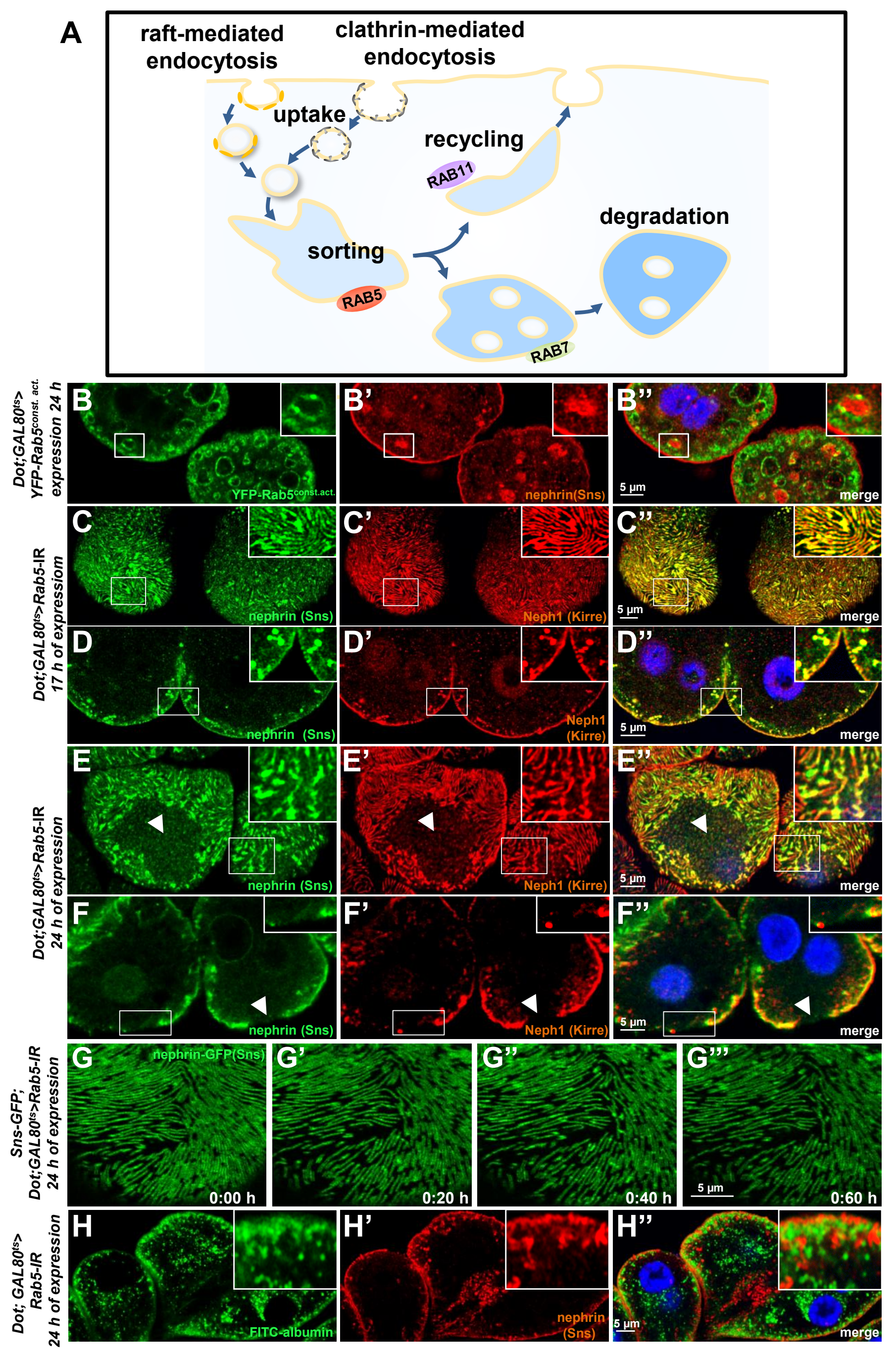

Fig. 3 
bioRxiv preprint doi: https://doi.org/10.1101/2022.03.01.482547; this version posted March 2, 2022. The copyright holder for this preprint (which was not certified by peer review) is the author/funder. All rights reserved. No reuse allowed without permission.

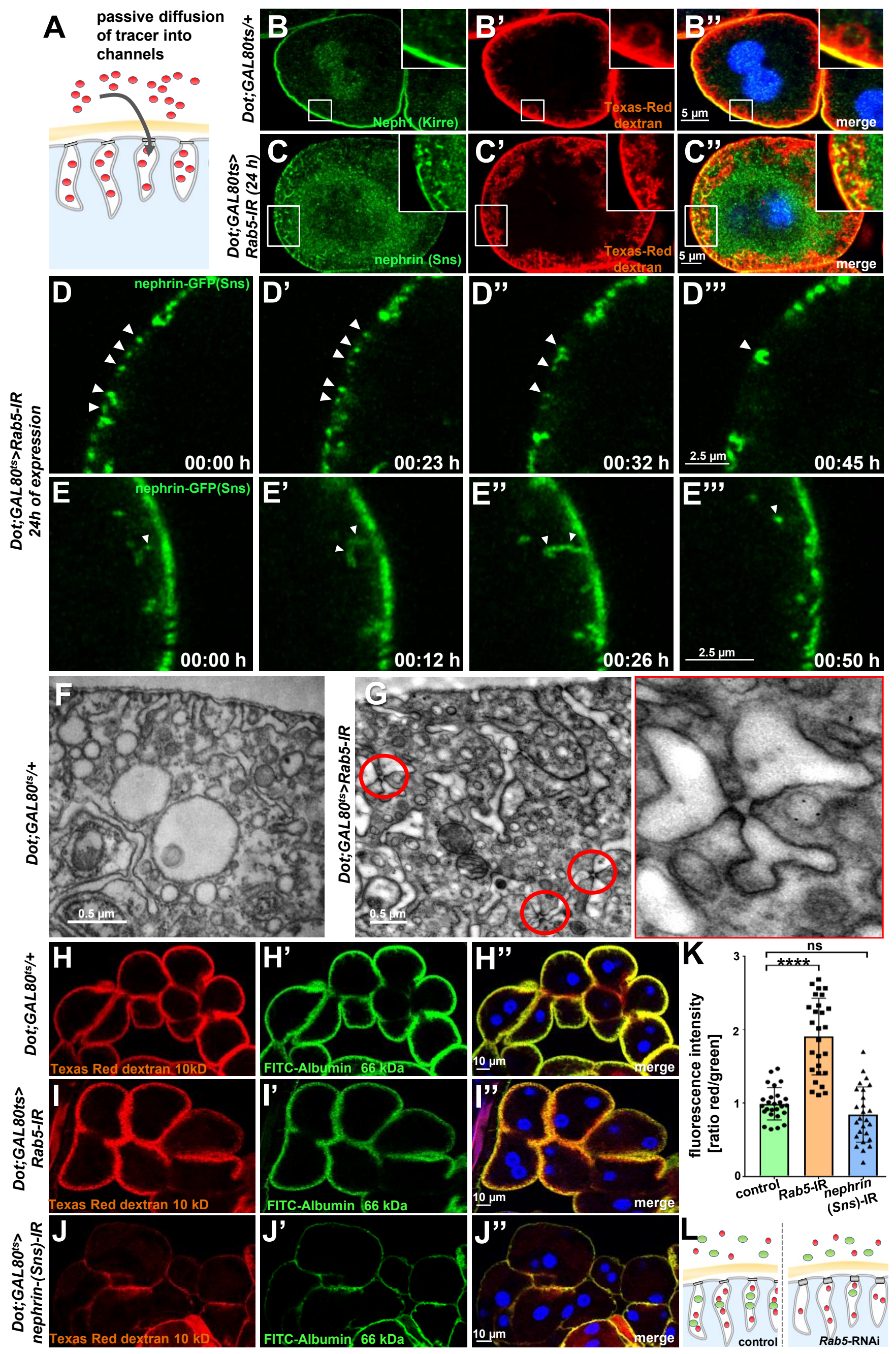

Fig. 4 
bioRxiv preprint doi: https://doi.org/10.1101/2022.03.01.482547; this version posted March 2, 2022. The copyright holder for this preprint (which was not certified by peer review) is the author/funder. All rights reserved. No reuse allowed without permission.
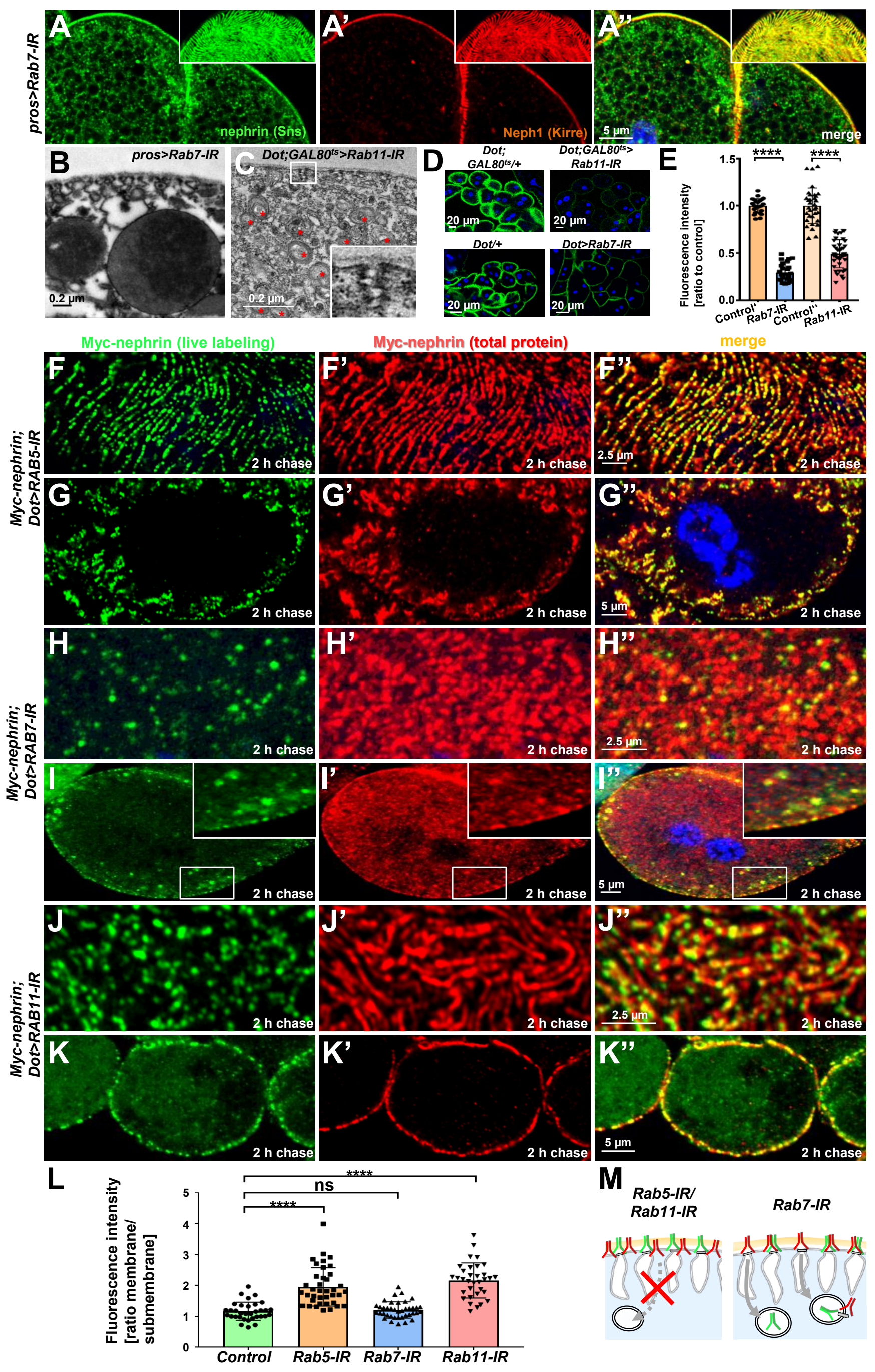

Fig. 5 


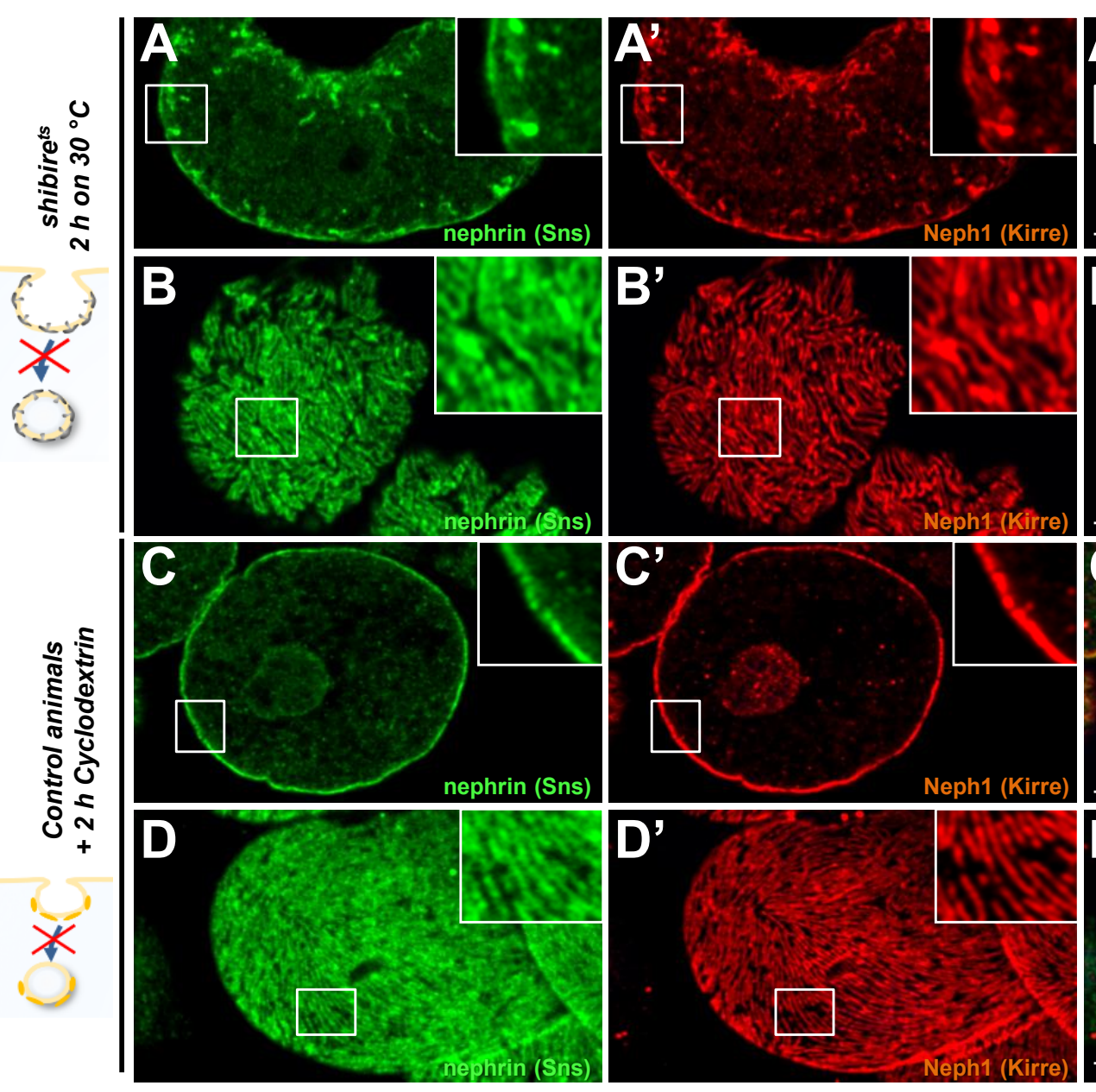

Myc-nephrin
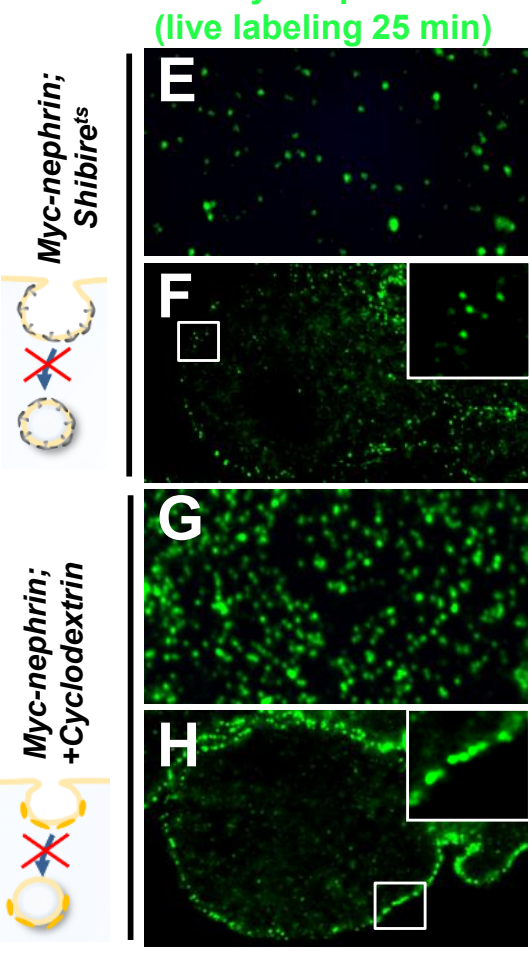

Myc-nephrin

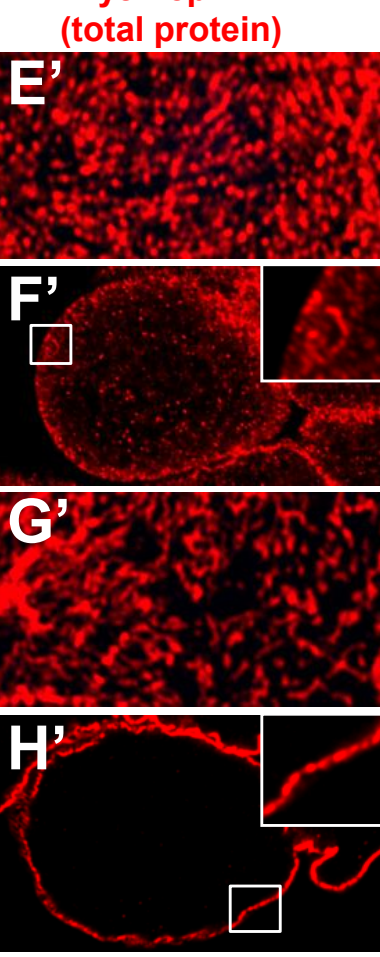

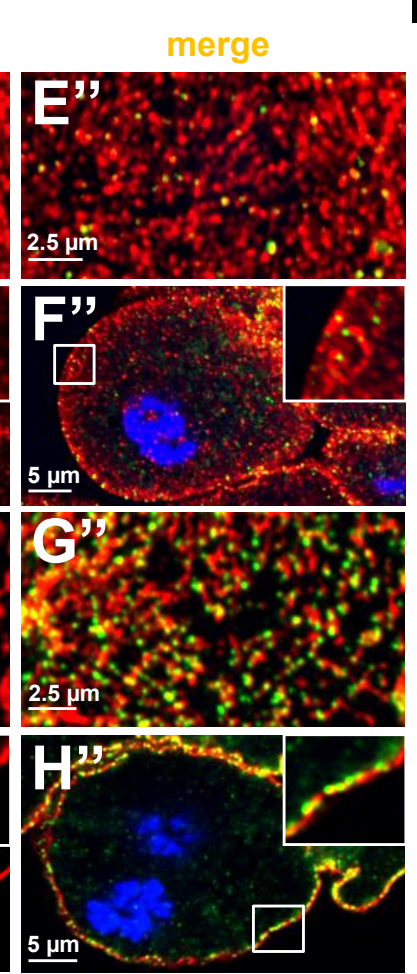

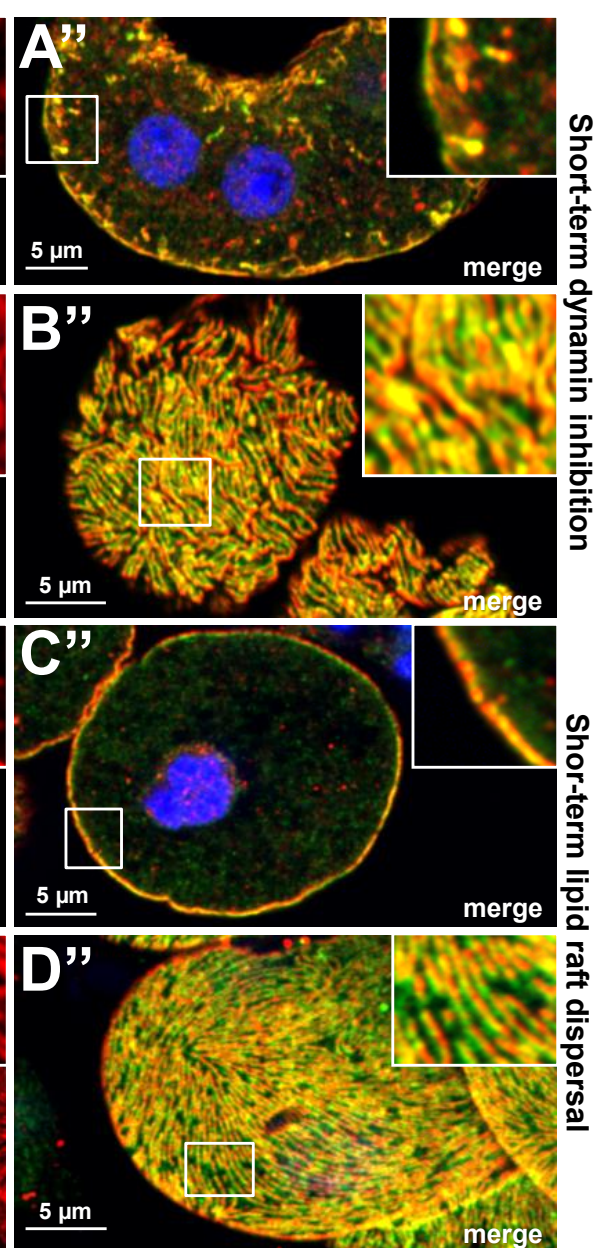

I

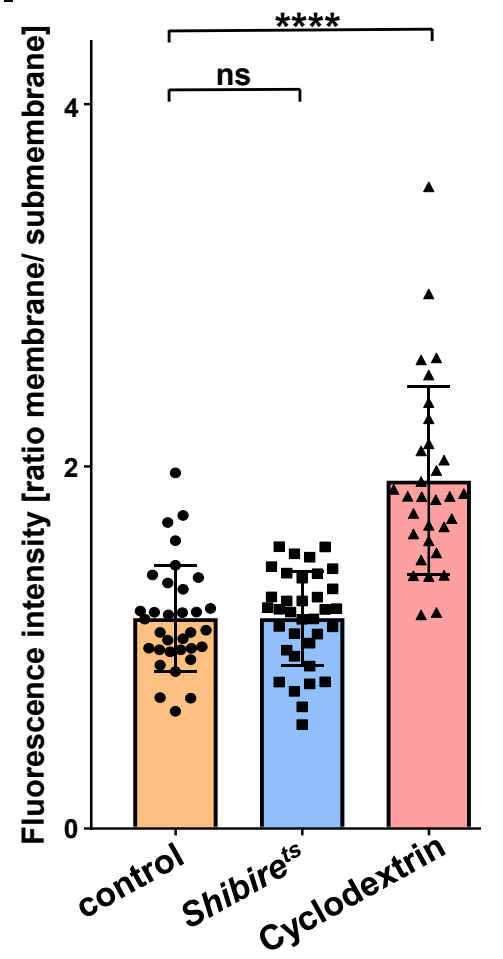

Fig. 6 

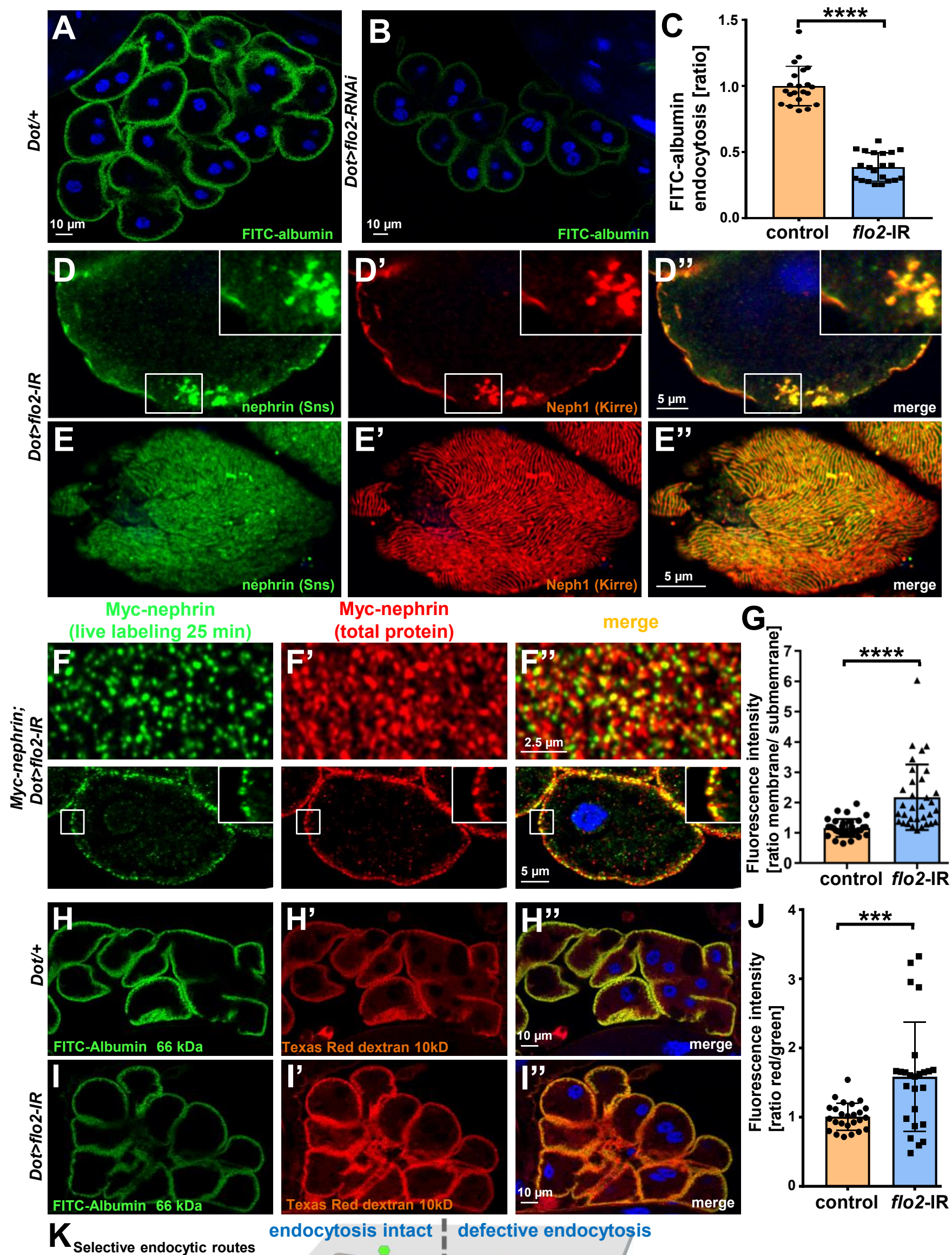

endocytosis intact defective endocytosis

converge for sorting, followed by recycling.

Flotillin2(raft)-mediated endocytosis of nephrin occurs at the slit diaphragm.

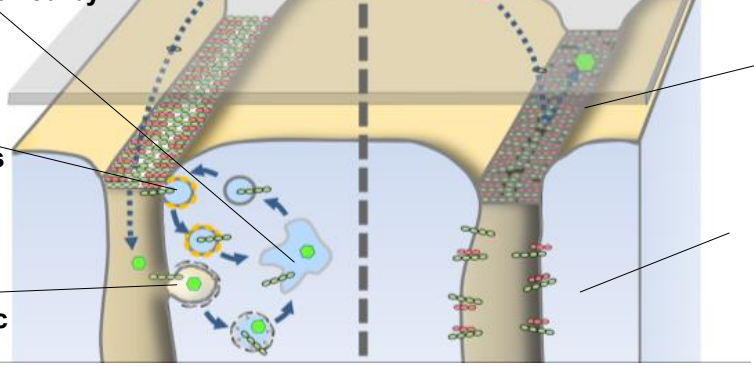

Lack of raft-mediated turnover results in filter clogging.

Lack of clathrin-mediated

endocytosis entails lateral diffusion

of slit diaphragm protein. 


\section{TABLE OF SUPPLEMENTAL CONTENT}

Supplemental Figure 1: Validation of nephrin-GFP and additional time points for disruption and reassembly of slit diaphragms.

Supplemental Figure 2: Validation of Myc-nephrin

Supplemental Figure 3: Validation and control experiments for loss-of-function of Rab5.

Supplemental Figure 4: Channel assay reveals loss of invaginations upon silencing of nephrin and impaired slit diaphragm passage upon silencing of Rab5.

Supplemental Figure 5: Validation and controls for Rab7 and Rab11.

Supplemental Figure 6: Additional images for live antibody labelling and validation and controls for Shibirets.

Supplemental Figure 7: Silencing flotillin2 using a second RNAi line confirms reduced FITCalbumin uptake and altered permeability of the filtration barrier.

Supplemental Video 1: Nephrin-GFP in larval nephrocyte tangential section

Supplemental Video 2: Nephrin-GFP in larval nephrocyte cross section.

Supplemental Video 3: Nephrin-GFP in larval nephrocyte upon transient nephrin silencing. Supplemental Video 4: Nephrin-GFP in larval nephrocyte upon acute Rab5 silencing (surface).

Supplemental Video 5: Nephrin-GFP in larval nephrocyte upon acute Rab5 silencing (breakdown of slit diaphragms).

Supplemental Video 6: Nephrin-GFP in larval nephrocyte upon acute Rab5 silencing (lateral diffusion). 
bioRxiv preprint doi: https://doi.org/10.1101/2022.03.01.482547; this version posted March 2, 2022. The copyright holder for this preprint (which was not certified by peer review) is the author/funder. All rights reserved. No reuse allowed without permission.

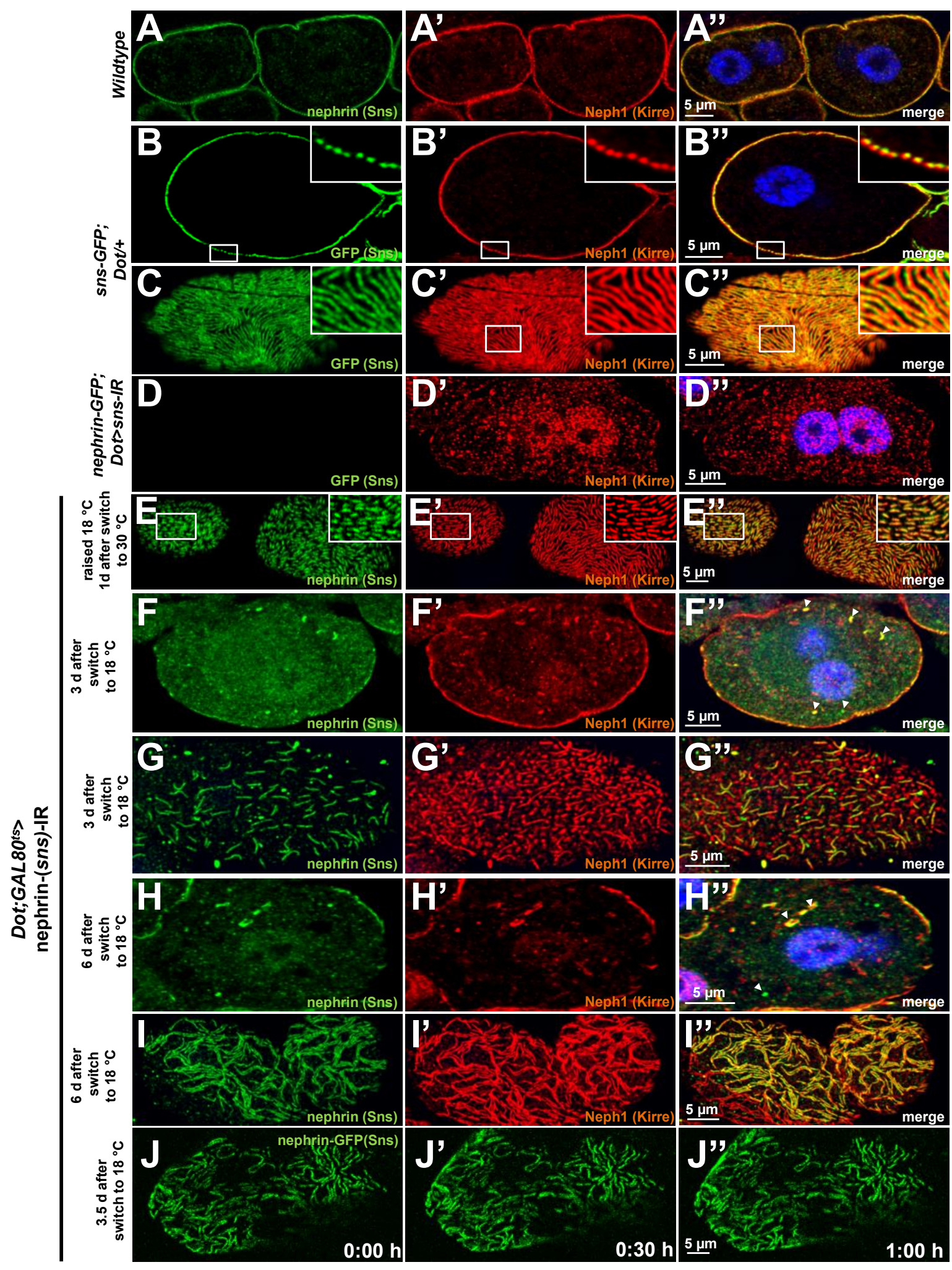

Supplemental Figure 1: Validation of nephrin-GFP and additional time points for disruption and reassembly of slit diaphragms.

(A) Cross section of a control garland cell nephrocyte (+/+) co-stained for nephrin (Sns) and Neph1 (Kirre). Nuclei are marked by Hoechst 33342 in blue here and throughout the figure.

(B-D') GFP-derived signal of nephrocytes from animals carrying genomic nephrin-GFP homozygously, matches the regular slit diaphragm pattern of endogenous Neph1 in cross sections (B-B') and tangential sections (C-C") but is abrogated upon silencing of fly nephrin (D-D").

(E-E') Lines of slit diaphragm proteins begin to shorten after $24 \mathrm{~h}$ of fly nephrin silencing.

(F-I') Fly nephrin is still strongly reduced three days after expression of RNAi against fly nephrin has been inhibited by a temperature shift to $18^{\circ} \mathrm{C}$ (F-F"). Nephrin (Sns) begins to return with lines elongating while fly Neph1 is resides mostly in punctae after four days (G-G"). Slit diaphragms cover most of the cell surface in clustered lines six days after the temperature shift ( $\mathrm{H}-$-').

(J-J'") Live-cell imaging of nephrin-GFP nephrocytes 3.5 days after transient silencing of nephrin shows reconstitution occurs at a slow rate. 
A
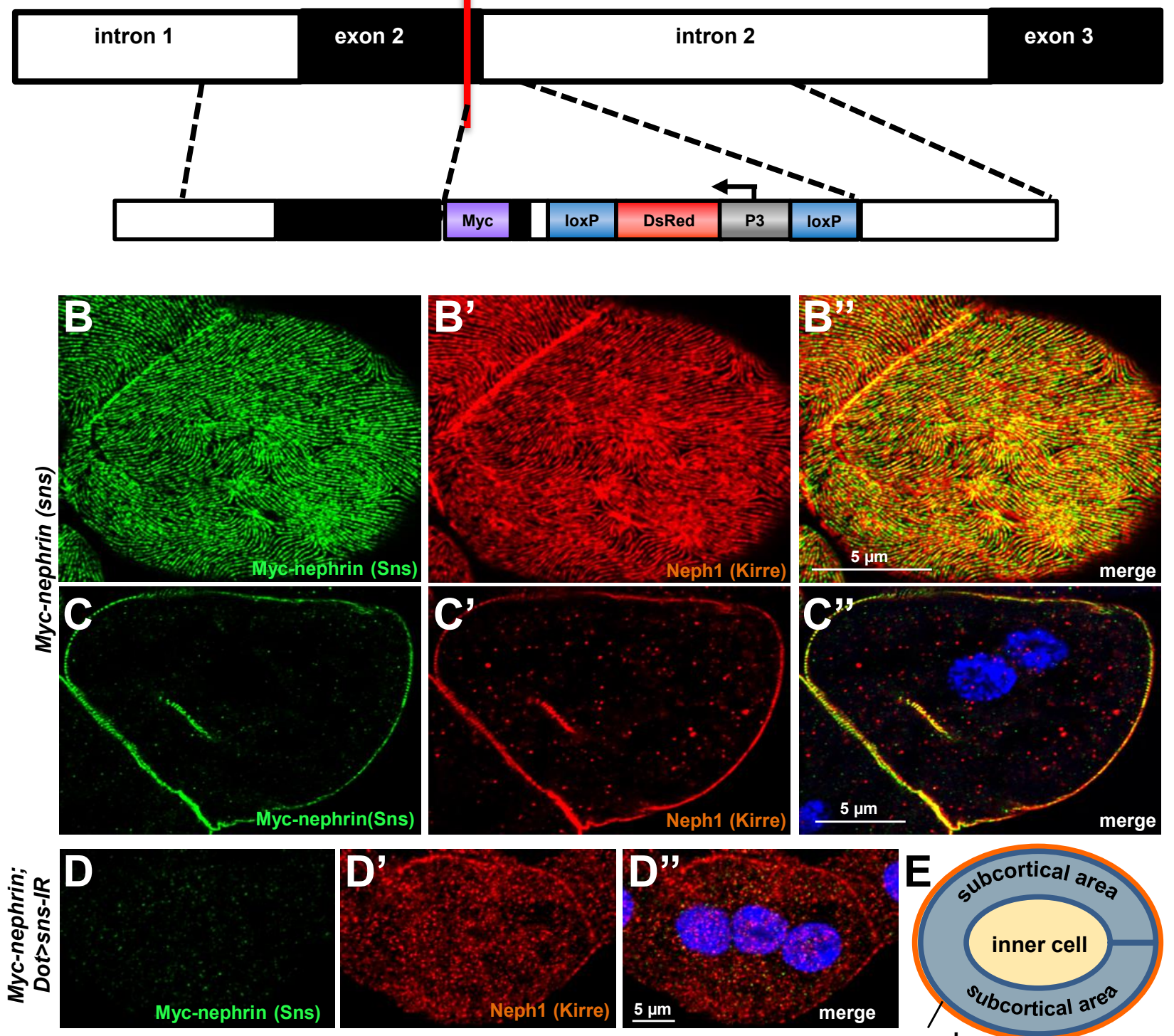

\section{Supplemental Figure 2: Validation of Myc-nephrin.}

(A) Shown is a schematic that indicates the genome editing strategy of introducing a myc-tag into the extracellular domain of sns, the fly nephrin. While myc is targeted to the border of exon 2, a marker (in reverse orientation) is inserted into the flanking intron. The marker expresses Dsred under control of the P3 promoter for identification of genome edited flies, but is removable by flanking loxP sites. (B-C') Shown are a tangential section (B-B") and a cross section (C-C") of a garland cell nephrocyte that carries Myc-tag in frame within the locus of fly nephrin, stained for Myc and Neph1 (Kirre). The Myc staining reveals a highly specific staining in a typical fingerprint-like pattern and colocalizes with endogenous fly Neph1. Nuclei are marked by Hoechst 33342 in blue here and throughout the figure. (D-D') Silencing fly nephrin abrogates the specific signal from Myc-staining, confirming that the Myc staining indeed reflects endogenously expressed Myc-nephrin.

(E) Schematic drawing of the areas used for the quantitation in Figure 2G and $5 \mathrm{~L}$ (membrane= orange and subcortical area= blue). 
bioRxiv preprint doi: https://doi.org/10.1101/2022.03.01.482547; this version posted March 2, 2022. The copyright holder for this preprint (which was not certified by peer review) is the author/funder. All rights reserved. No reuse allowed without permission.

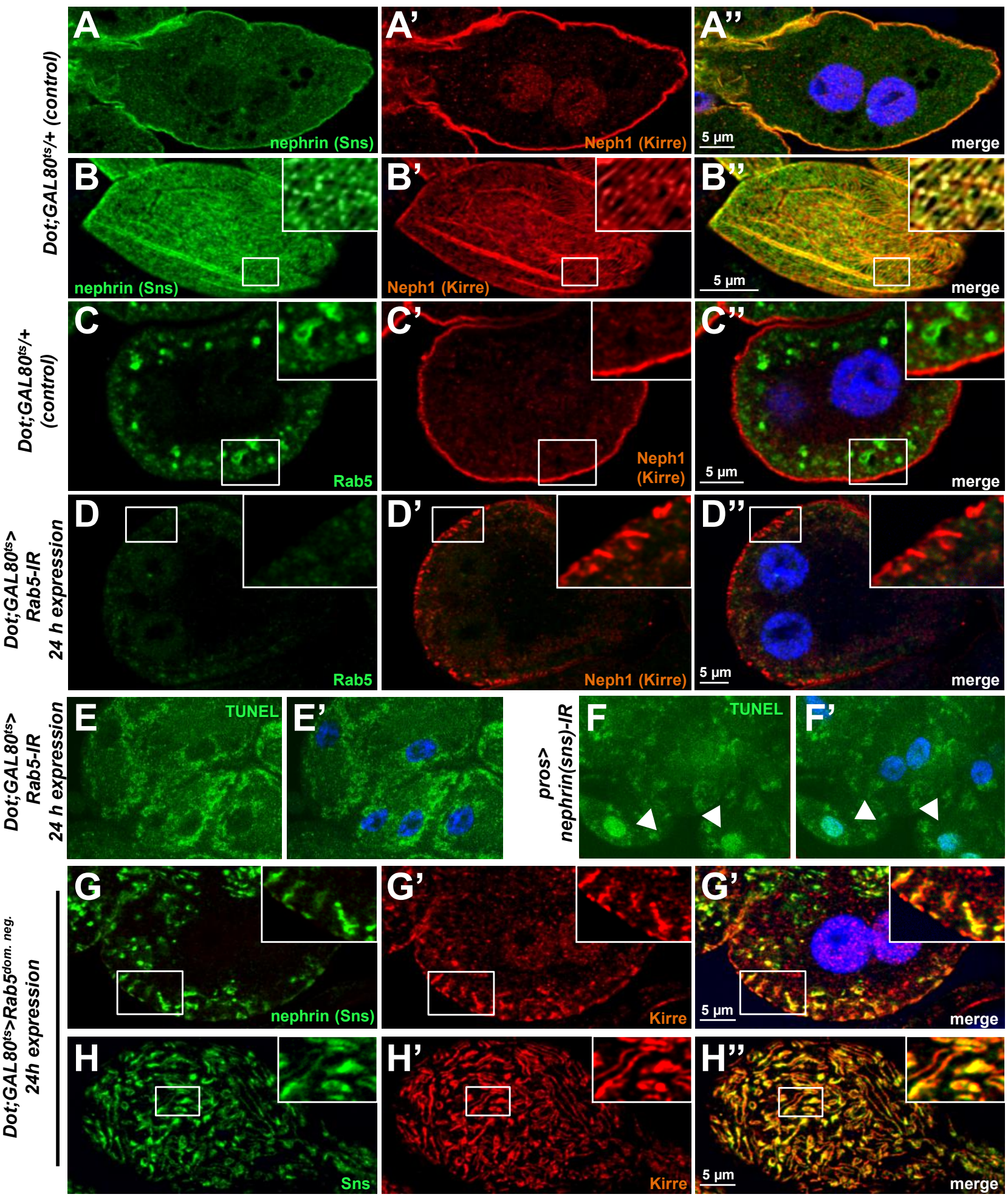

\section{Supplemental Figure 3: Validation and controls for loss-of-function of Rab5.}

(A-B') Shown are a cross section (A-A") and a tangential section (B-B") of a garland cell nephrocyte that expresses GAL80ts alone, stained for Nephrin (Sns) and Neph1 (Kirre). Nuclei are marked by Hoechst 33342 in blue here and throughout the figure.

(C-D') Rab5 stains in small vesicles at the cell periphery in control nephrocytes (C-C'). Silencing Rab5 strongly diminishes the Rab5 signal and fly Neph1 reveals mislocalization. This indicates that short term silencing is sufficient for a significant knockdown of Rab5.

(E-F') Nephrocytes expressing Rab5-RNAi for $24 \mathrm{~h}$ were subject to TUNEL staining but no specific signal from the nuclei is observed (E, compare to Hoechst 33342 in E'), indicating that cells are not apoptotic. In contrast, when silencing fly nephrin as a positive control, we observed appearance of TUNEL-positive cells (F-F').

(G-H') nephrocytes with short-term expresison of a dominant negative Rab5 for 24 h show ectopic slit diaphragm protein below the surface in cross sections (G-G") and blurry and confluent lines of slit diaphragm protein in tangential sections $\left(\mathrm{H}-\mathrm{H}^{\prime \prime}\right)$. 

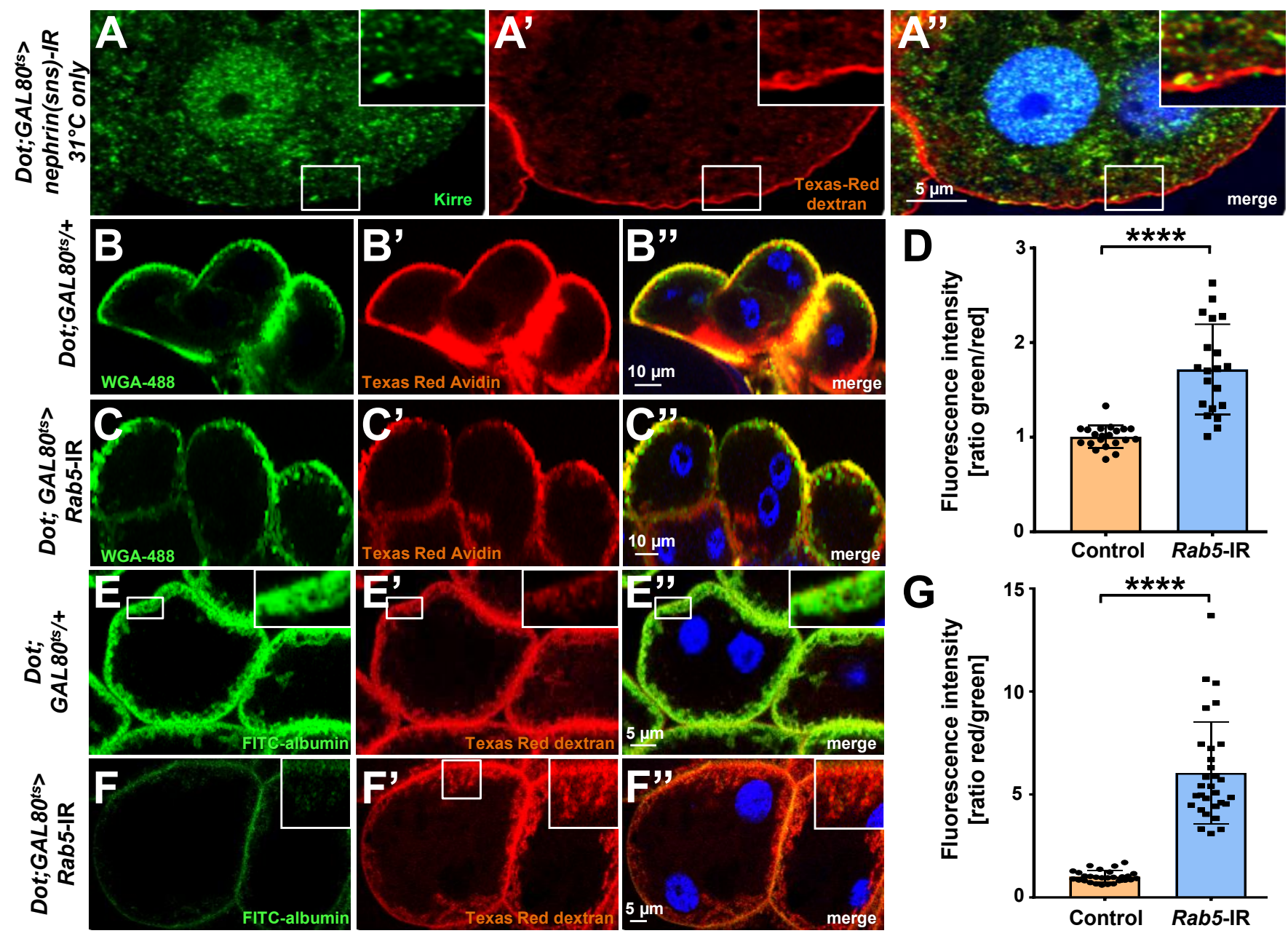

Supplemental Figure 4: Channel diffusion assay reveals loss of invaginations upon silencing of nephrin and impaired slit diaphragm passage upon silencing of Rab5.

(A-A') Shown is a cross section (A-A") of a garland cell nephrocyte subject to the channel diffusion assay. Texas-Red-dextran does not penetrate deeper into the cell when channels are abrogated by expression nephrin (Sns)-RNAi, supporting that the signal is specific for the membrane invaginations called labyrinthine channels. Nuclei are marked by Hoechst 33342 in blue here and throughout the figure.

(B-C') Confocal microscopy image of nephrocytes after simultaneous uptake of Alexa488 wheat germ agglutinin (38 kDa, green) and the larger tracer Texas Red-avidin (66 kDa) for control nephrocytes (B) and after silencing Rab5 (C), which has a weaker impact on uptake of the smaller tracer.

(D) Quantitation of fluorescence intensity expressed as a ratio of WGA-488/Texas-Red-Avidin (small/large tracer) confirms disproportionate reduction for the larger tracer upon expression of Rab5RNAi (Mean \pm standard deviation $n=7, \mathrm{P}<0.0001$ for Rab5-RNAi).

(E-F') The channel assay reveals a greater reduction in FITC-albumin penetration into channels compared to the smaller Texas-Red-dextran (10 kDa) for silencing of Rab5 (F-F") compared to the control (E-E"').

(G) Quantitation of fluorescence intensity expressed as a ratio Texas-Red-Dextran/FITC-albumin (small/large tracer) further confirms the described disbalanced reduction for the larger tracer for Rab5-RNAi (Mean \pm standard deviation, $\mathrm{n}=9$, $\mathrm{P}<0.0001$ for Rab5-RNAi). 

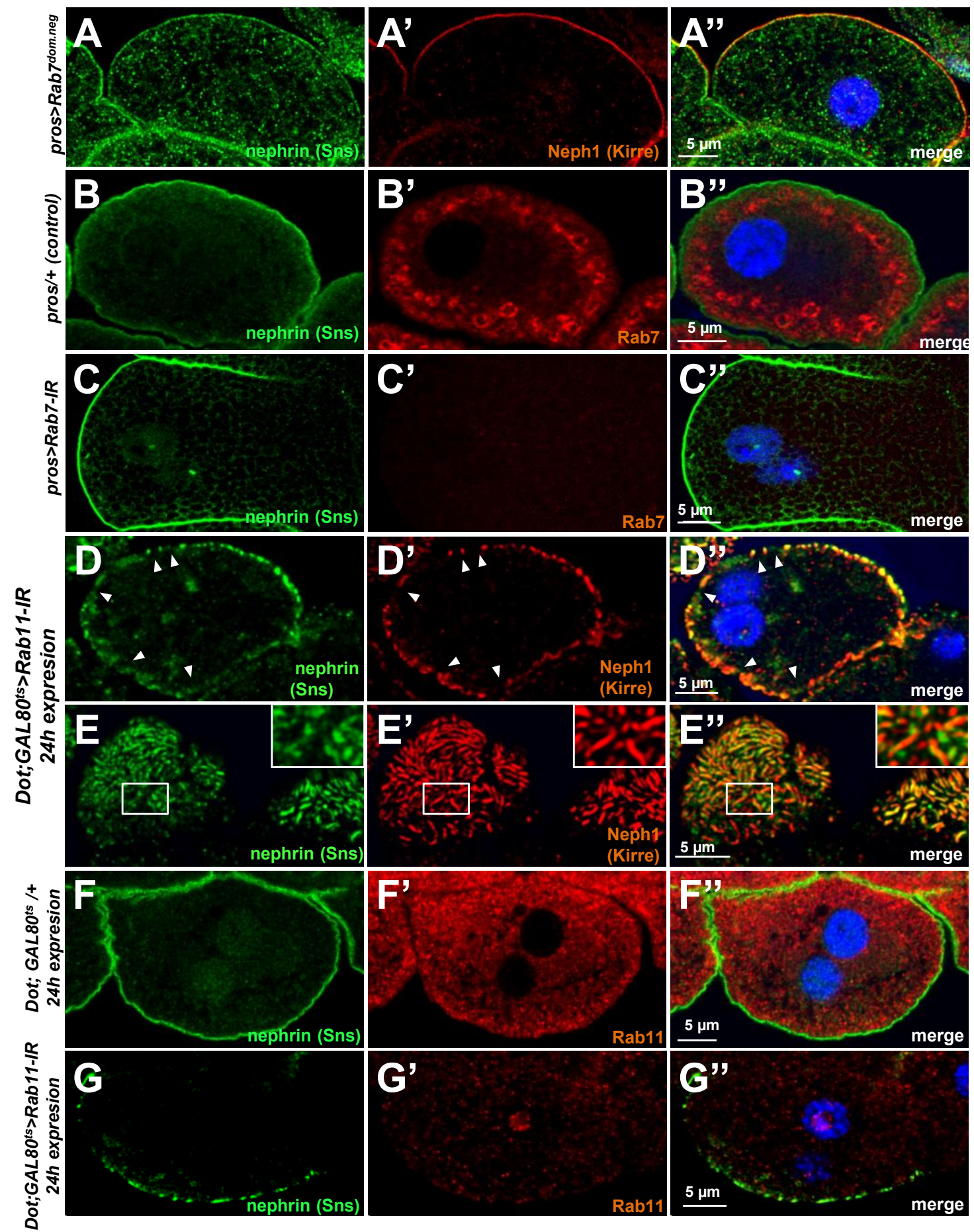

\section{Supplemental Figure 5: Validation and controls for Rab7 and Rab11.}

(A-A") Slit diaphragms are formed regularly upon expression of dominant negative Rab7, while nephrin accumulates diffusely in the cell. Fly Neph1 is less affected than fly nephrin upon silencing of Rab7.

(B-C') Control nephrocytes expressing prospero-GAL4 alone (B-B") show the regular staining pattern of fly nephrin (Sns) and Rab7. Signal of the Rab7 antibody is lost upon expression of Rab7-RNAi (C-C"). (D-E') Acute silencing of Rab11 for $24 \mathrm{~h}$ in nephrocytes results in coarser, wider spaced dots in crosssections (D-D") matching wider gaps between the lines of slit diaphragm proteins in tangential sections (E-E"). Slit diaphragm proteins may occasionally occur independently from each other (inset in E-E"). (F-G') Short term expression of Rab11-RNAi strongly diminishes the signal derived the from an antibody raised against human Rab11 (compare F-F" to G-G") suggesting an efficient knockdown. 

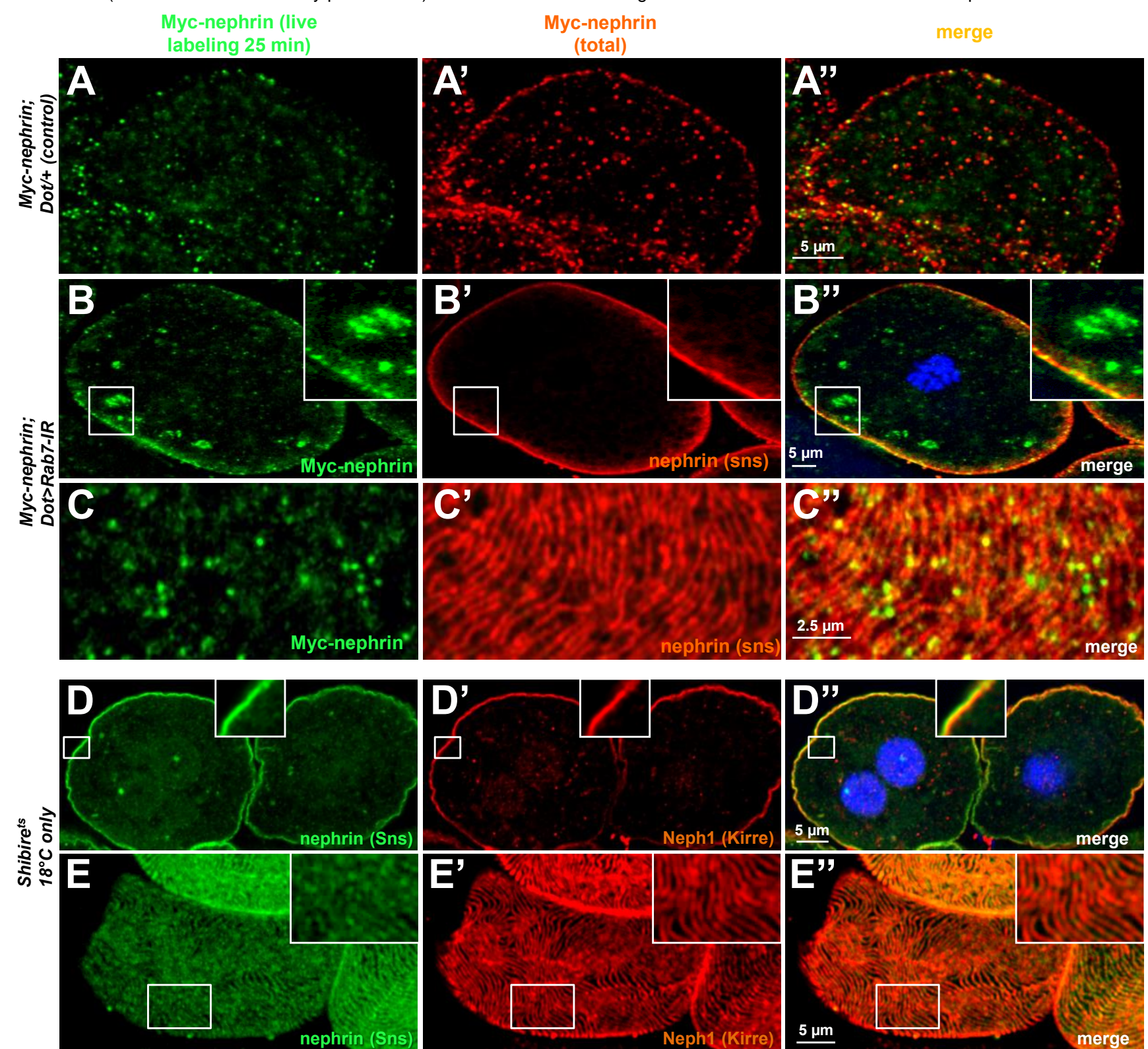

Myc-nephrin

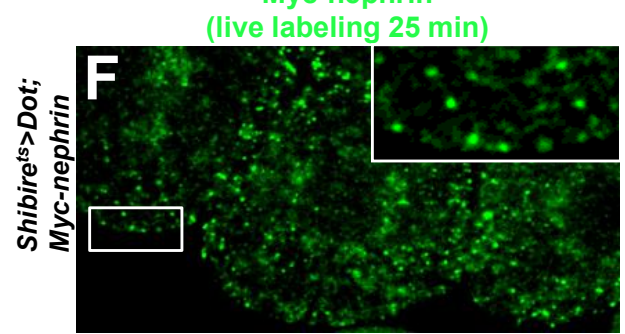

Kirre

(Neph1)

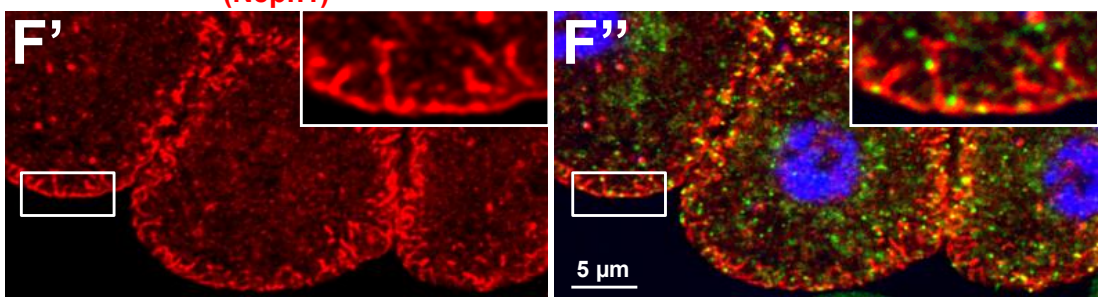

Supplemental Figure 6: Additional images for live antibody labeling and validation and controls for Shibirets.

(A-A") Confocal images of control nephrocytes that express Myc-nephrin heterozygously show complete turnover, not distinguishable from cells that carry the genomed edited locus homozygously (compare to Figure 2).

(B-C') Confocal images of nephrocyte expressing Rab7-RNAi after live antibody labeling show subcortical vesicles that exclusively stain for the live labeled antibody (green) but not for nephrin staining, suggesting they contain antibody that is no longer coupled to nephrin (B-B"). Tangential sections from the same cell confirm undisturbed nephrin turnover as the live labeled antibody is removed from the surface (C-C"). Nuclei are marked by Hoechst 33342 in blue here and throughout the figure.

(D-E') Nephrocytes carrying the temperature sensitive allele of shibire show a regular staining pattern at a lower temperature at which the protein remains functional.

(F-F') A chase time of 120 min after live antibody labeling is shown for the temperature sensitive allele of shibire with Kirre co-staining. Most of the live labeled nephrin is removed from the cell surface while Kirre indicates a severe mislocalization. 
bioRxiv preprint doi: https://doi.org/10.1101/2022.03.01.482547; this version posted March 2, 2022. The copyright holder for this preprint (which was not certified by peer review) is the author/funder. All rights reserved. No reuse allowed without permission.
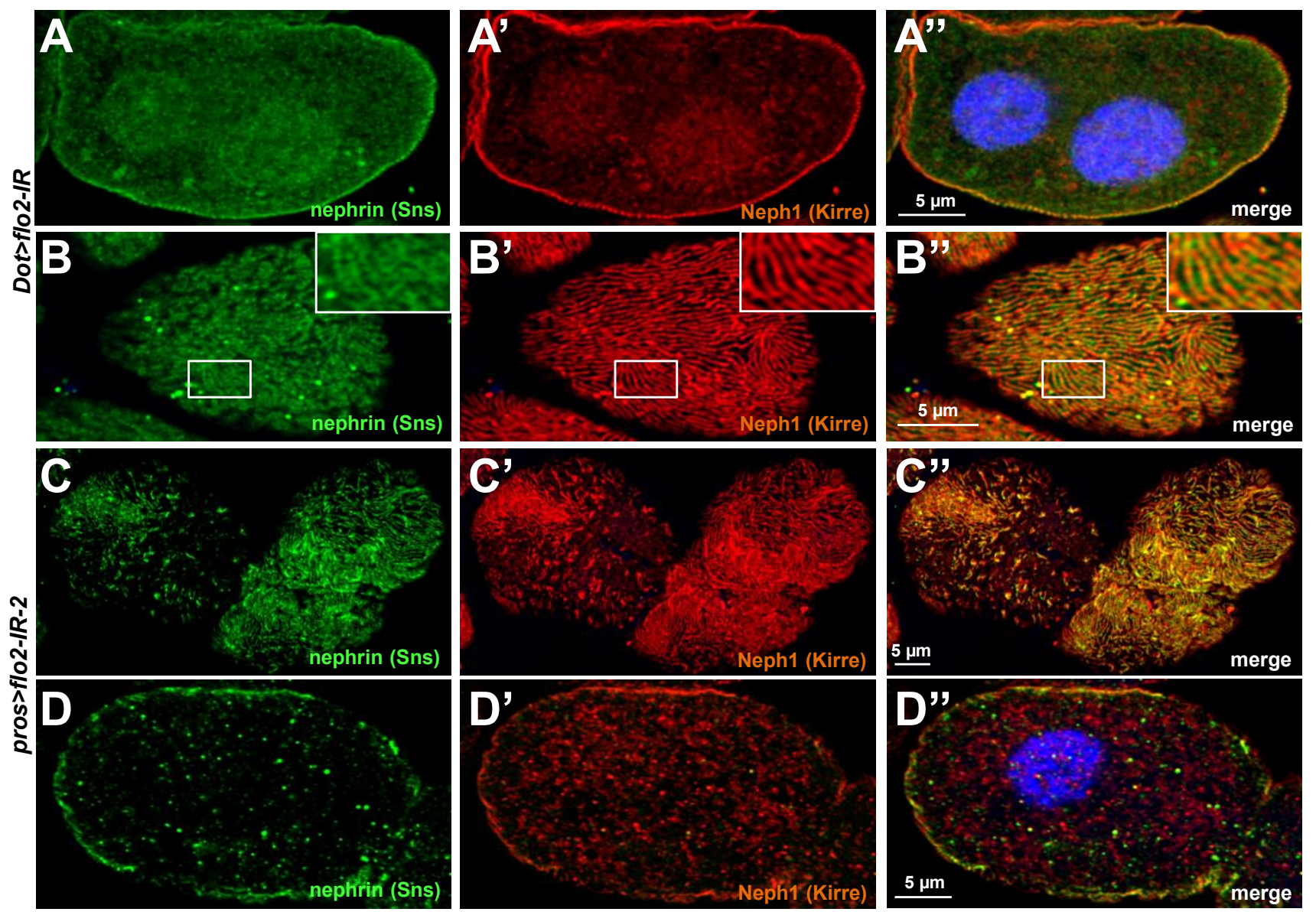
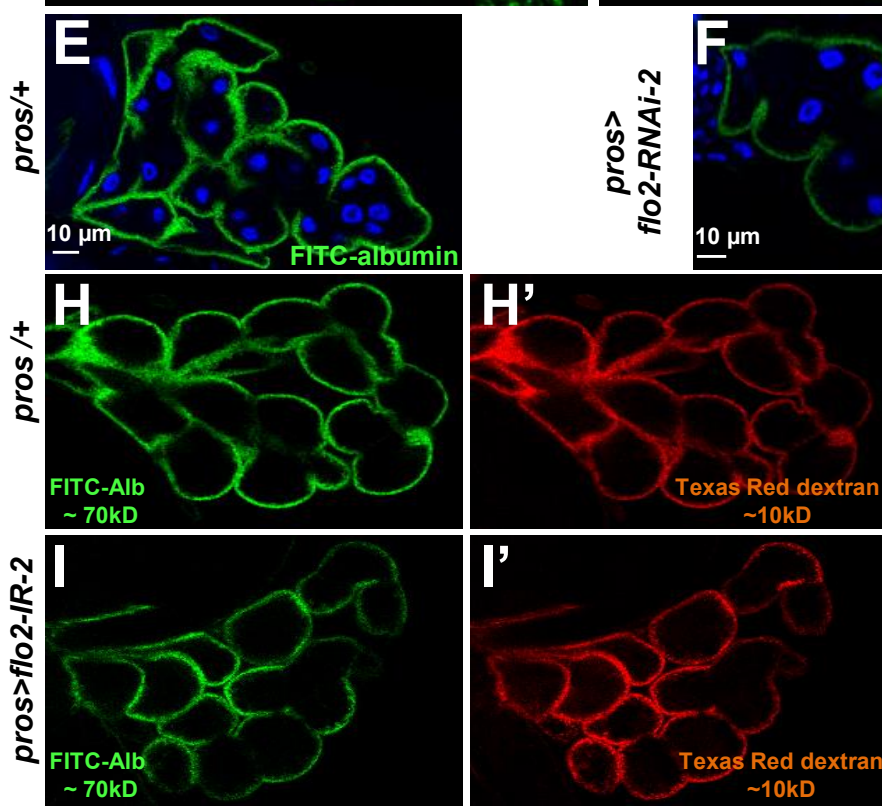

FITC-albumin
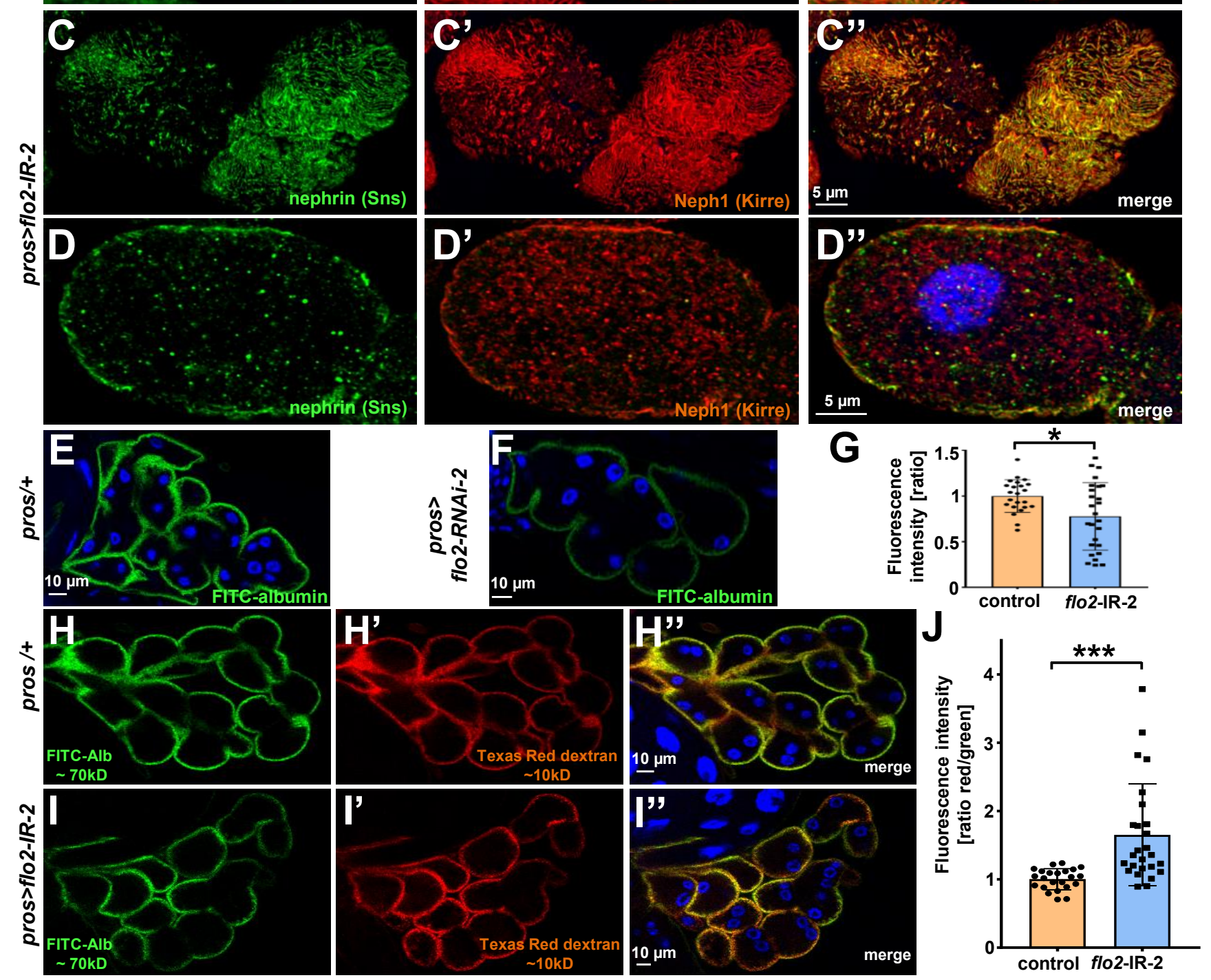

\section{Supplemental Figure 7: Silencing flotillin2 using a second RNAi line confirms reduced FITC-albumin uptake} and altered permeability of the filtration barrier.

(A-D) Silencing of flo2 may result in regular nephrin/Neph1 staining (A-B, using flo2-RNAi 1) or localized breakdown of slit diaphragm (C-D, using flo2-RNAi 2). Nuclei are marked by Hoechst 33342 in blue here and throughout the figure.

(E-F) Confocal microscopy image of nephrocytes after uptake of FITC-albumin show impaired uptake of nephrocytes expressing flo2-RNAi-2 (F) compared to control cells (E).

(G) Quantitation of results from $(E-F)$ in ratio to a control experiment performed in parallel ( $n=8-9$ per genotype, $\mathrm{P}<0.05$ for flo2-RNAi-2).

(H-I') Confocal microscopy image of nephrocytes after simultaneous uptake of FITC-albumin (66 kDa, green) and the smaller endocytic tracer Texas-Red-dextran $(10 \mathrm{kDa})$ are shown. Silencing of flo 2 causes a relatively stronger decrease in the uptake of the larger tracer FITC-albumin compared to smaller Texas-Red-dextran.

(J) Quantitation of fluorescence intensity expressed as a ratio of Texas-Red-Dextran/FITC-albumin (small/large tracer) confirms a disproportionate reduction for the larger tracer for flo2-RNAi-2 ( $n=8-9, P<0.001$ for flo2-RNAi-2). 